\title{
A COUPLED MHD-MONTE CARLO TRANSPORT MODEL FOR DENSE PLASMAS
}

W1lliam Paul Chandler

(Ph. D. Thesis)

June, 1975

Prepared for U.S. Energy Research \& Development Administration under contract No. W-7405-Eng-48

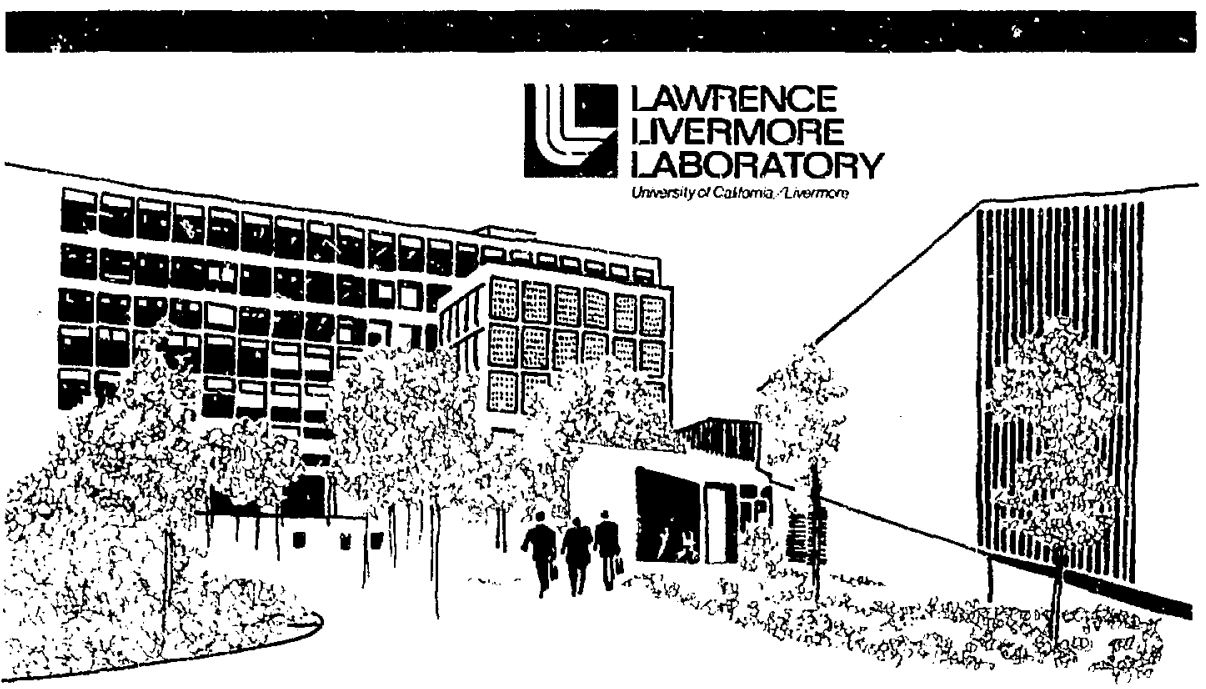




\section{Notict:}

"This report was prepiated as an accoumt "I wotk spon:ared by the Uniled States Cinvernment. Netilier the United States not the United Slates Hargy Heseasch \& Developmeni Administratiun, hisr atis of their employess, nor any of their contraclors, subcontractnrs, of their empluyes. makes asy warranty. expiess of implied, of gwutnes any legal liability of responsihility fur the sccuracy, completeness or usefulnuss of ans information. epparatus, producl or pacass disclesed, or represents that its use would nirt inferinge uniwaly owned riglits."

Printed in the United States of America Available from

National Technical Information Service U.S. Department of Commerce 5285 Port Royal Road Springfield, Virginia 22151 Price: Printed Copy \$_; Microfiche $\$ 2.25$

$$
\begin{gathered}
* \text { Pages } \\
\frac{*-50}{51-150} \\
151-325 \\
326-500 \\
501-1000
\end{gathered}
$$

NTIS

Selling Price

$\$ 4.00$

$\$ 5.45$

$\$ 7.60$

$\$ 10.60$

$\$ 13.60$ 


\title{
㢟 \\ LAWPENCE LNERMORE LABORATORY \\ Universily of Celtoria Lnermoro.Caltornia 94550
}

\section{LICRI. -52009 \\ A COUPLED MHD-MONTE CARLO TRANSPORT MODEL FOR DENSE PLASMAS}

\author{
William Paul Chandler \\ (Ph. is. Thesis) \\ MS. Date: June, 1975
}

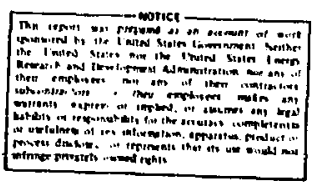


TABLE: (H) TONTESTS

Pasce

ABSTknt $\ldots \ldots \ldots \ldots \ldots \ldots \ldots \ldots \ldots \ldots \ldots \ldots \ldots \ldots \ldots \ldots \ldots \ldots \ldots \ldots \ldots \ldots \ldots$

1. Inthublicin $\ldots \ldots \ldots \ldots \ldots \ldots \ldots \ldots \ldots \ldots \ldots \ldots \ldots \ldots \ldots \ldots \ldots \ldots \ldots \ldots \ldots$

2. NHD NOULL $\ldots \ldots \ldots \ldots \ldots \ldots \ldots \ldots \ldots \ldots \ldots \ldots \ldots \ldots \ldots \ldots \ldots \ldots \ldots \ldots \ldots$ 7

2.1 Conservation lquat lons $\ldots \ldots \ldots \ldots \ldots \ldots \ldots \ldots \ldots \ldots \ldots \ldots \ldots \ldots$

2.2 Defference liquate lons; Momentur: $\ldots \ldots \ldots \ldots \ldots \ldots \ldots \ldots \ldots \ldots 13$

2.1 Defferenct Equaltions: Vnergy ................... 19

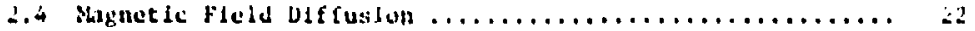

$\therefore 5$ loule Heatiny. ............................ 29

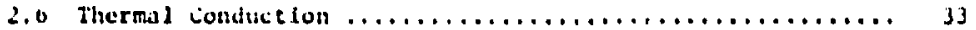

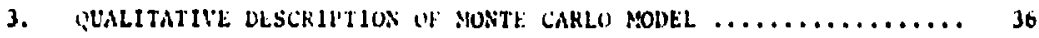

3.1 Integration ot tor untz fores ................... 40

3.2 Integration 4 stall Angle colltsion Term ........... is

J.3 Integrat lon of Large Anf: le collisten Term ............ 49

3.2 Momentin Conservation ....................... 53

3.5 Nonte Gir to Current ......................... 59

4. Cuscllsion $\ldots \ldots \ldots \ldots \ldots \ldots \ldots \ldots \ldots \ldots \ldots \ldots \ldots \ldots \ldots \ldots \ldots \ldots \ldots \ldots$

5. NPENDICES

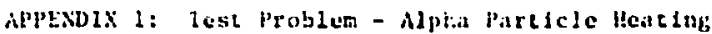

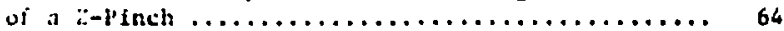

APENind 2: Iranspore coeffiedents $\ldots \ldots \ldots \ldots \ldots \ldots \ldots \ldots \ldots \ldots$

APplidix 3: Artiflcial viscosity ................

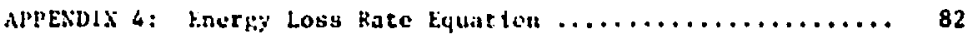

Mplexblx 5: Tracking; in a 20 l.tkranklan Cylindrical Mesh ..... 85

APPENDIX 6: Yonte carlo Technfques $\ldots \ldots \ldots \ldots \ldots \ldots \ldots \ldots \ldots$. 92

APpENULx 7: Energetic Ion Scurce iof Stonte carlo :odel ...... 94

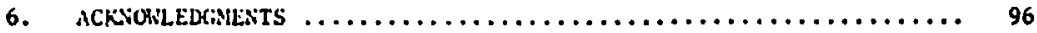

7. REFERENCES $\ldots \ldots \ldots \ldots \ldots \ldots \ldots \ldots \ldots \ldots \ldots \ldots \ldots \ldots \ldots \ldots \ldots \ldots \ldots \ldots \ldots \ldots$ 
A COUPLED SHD-NOXTE CARLU TKANSPOKT MODEL FOR DENSE PLASMAS

\author{
hillidit P. Chandler \\ University of California, Lithrence Livermore Laboratory \\ Livermore, Cd 94550 \\ Jus11., $19 \% 5$
}

\title{
ABSTKLT
}

A two-dimensfonal. tho fluld model of the MID equations las been coupled to a Monte Carlo transport model of hiph unergy, non-Maxwelizan long. The MHD part of the tiodel assumes complete fonlzation and includes a perfect gas law for a scalar pressure, a tensor artificial viscosity, electron and ion thermal conductan, electron-ion coupling, and a radiation loss cerm. A simple ohm's Law is used with a $B_{t}$ mignetic field. The NaD equations were solved in Lagrangian coordinates. The conservation equatiens were differenced explicitly and the diffusion-type equations implicitly using the splitting technique. The Nonte Carlo medel solves the equation of motion for high energy lons, moving through and suffering small and large angle collistons with the fluid Maxwellian plasma. The source of high energy lons is the thermonuclear reactions of the hydrogen isotopes, or th may be an externally injected beam of neutralized Ions. In addition to using the usual Maxwell averaged thermonuclear cross sections for calculating the number of reactions taking place within the Noxwellian plasma, the hlgh energy lons may suffer colldsions resulting in a reaction. In the Monte Carlo model all neutrons are assumed to escape, and all energetic lons of $Z \leqq 2$ are lollowed. 
This model cin be used co calculate the effect of a non-Maxwellian species interacting with a fluid species and is particularly useful in calculating the spatial variatlons of the energy depostctons to a Maxwellian fluid from a high energy nun-Maxwellian spectes. As a demonstration, the model was used to calculate the alpha particle heating of a very dense gasembedded 2-pinch. Heating itom 10 to $45 \mathrm{keV}$ is primarily due to the alpha heating, and consequently the pinch popands. The results of thisi calculation show that the alpha patticle energy depositions aze in falr agreenuth wth previous calculacluns, but that anergy losges due to drift are lower than expected. The difforunces can be attrlbuted to thic nonlocal elergy depositions of the alpha particles. 


\section{INTRODLCTION}

Computer codes and models that have been developed for the study of plasma physics related to the controlled fusion program have primarily been of three types: ${ }^{1}$

1. the cluid MHD model;

2. the partele models; and

3. the kinetic equation modeds.

The fluid MHi, model is computatiunally faster, but is generally limited to dense collition doninated fluids, or when the Larmor orbits of the fluld species is small compared to characteristic sizes of interest in the plasma fluid.

The particle codes give grcat detail at the expense of computer time and memory capacity, but generally don't consider the macroscopic fluld behavior of the plasma. "article codes usually do not consider collistons between the plasmil cumponents, but do solve the nonlinear vlasov equation and Maxwell's equations in a self consistent manner.

The kinetic eçuation codes solve the Vlasov and Fokker-Planck equations difecty by differencing of the transport equation or using Fourier cransform techniques. In nefther case is fluid behavior considered. The Fokker-Planck model is used mainly to calculate the time and velocity variations of a distribution function, but usualiy not the spatial varlations. 
The object of this study was to build a model and a code that would not only calculate the behavior of a dense fluid, but also the behavior of a species of high energy non-Maxwellian ions traveling through and interacting with the Naxwellian plasmi f!uld. The source of these energetiv lons within the fluid plasma may be the resuls of thermonucleat reactions and/or an infected beam of neutralized jons (a neutral cloud of lons and electrons). Computationally, the fluld bohuvior is modeled with the Mlid equations and the hiph energy non-Mixwellian fous with a Monte Carlo particle tracking and and colliston model. These two nodels are coupled through collislons in the Nonte $C_{\text {arlo }}$ routines, and surce terms in the NHD equation:.

In other words, the code does the follnwing physics. The alld model is a two-dimensional (cylindrical - $k, 2$ ) Lagrangian two fluid morlel. There is a Maxwelizan electron fluid at semperature $i_{e}$ and a Maxwellian Ion fluid at temperature $T_{i}$. The fon fluid tay be composej of all the isotopes of hydrogen and helium, but they are all assumed to be at the temperature $T_{1}$. A perfect gas equation of state 1 s used. The $f 1: 4 d$ equations were explicitly differenced in a maner similar to ,chulz, ${ }^{2,3}$ with his scheme for a tensur artificial viscosity. For simplicity, anly the $B_{Q}(R, Z)$ component of the magnetic field was programed, alinough the other components $\left(B_{R}, B_{Z}\right)$ could be added to the model. A simple Olum's Law was also assumed. The time derivatjue of the magnetic field was solved implicitly, since the approprlate Maxwell's equations were easily cast into a diffusion type equation. Fiectron and ion thermal conduction was also solved implicitly. The transport coefficients; $\kappa_{e}$ - electron thermel 
conductivity, ${ }_{i}$ - ion thermal conductivity, and $\sigma$ - electric conductivity were modeled after Braginskii; ${ }^{4} 1, t .$, they are functions of $B$ and $Z$ (atomic number) in addition to the usual variables $-n_{e}, n_{i}, T_{e}, T_{1}$. Because of the two temperature model, an olectron-ton coupling energy loss (gain) term was calculated. A radiation joss term due to bremsstrahlung was lacluded in the clectron enery cqualtion.

the source of high energy, non-Maxwellian lons was calculared from tho thermonuclear hydro!sen tsotope reaction rates ustag. the Naxwell averaged cross secilfons if irecue. 5 The high energy ton reaction products were then silpled in a Mutstu Carlo ranner, and were tranaported through the matnet 1 aed Maxiolldan lluid:i. The Monte Carlo particies nove through the fluid, under the action of the $\ddot{\forall} \times \vec{B}$ Lorentz force, and suffer srall and large dnyle collisions with phe iluid electrons and fons. The change in tho sionce carlo sundle's energy and momentum due so small angle collisfons it calcilated deterministically by integrating an energy loss rate equation (dE/dt). The Monte Carlo bundles may also suffer a large any:le scatter or an in-ilight thermunuclear reaction. The rejatively unimportant large angle scatters and the more Important in-fight reactions were done it. the usual Nonte Carlo manner; 1.e., random number selection weighted against collision and reaction probabilities.

In other words, a Monte Carlc high energy ion bundle would be generated and would be moved ajong its $\vec{v} \times \vec{B}$ crbit for some predetermined time step. At tine end of the time step, the energy and momentum changes over that step aro calculated due to small angle collisions with electrons and ions. It is assumed that the time to equilibration is less than the 
time for a $90^{\circ}$ deflection, therefore there would be no deflection of the Nonte Carlo bundle due to small angle collisions. Now the probability of having a large angle scatter or in-flipht reactlon over chat path are calculated and compared to a randin number. If a collision uccurred, then It is assumed to take place at the end if the pach and the colitsion kfnentics art calsulated. If no colliston occurs, the tracking of the particle is conthued for another tine scep. Changes in the iluid's inergy and momentum du' to th' fonce carlo bundles dre tinen used as source cerms in the ener ${ }^{\prime} y$ and momentum equations in the shib section. See fig. 1 for a very simplified block diagram of the model. In Fis. 1 , $\vec{F}$ is the iluid velocity, $\vec{v}$ is the ronte carlu bundle velocity, she fluid density, $J_{M C}$ is the current due to the motion of the high energy Nonte Carlo bundles, and $T_{e}$ and $T_{i}$ are the electron and ion temperatures.

It should be noted that the model is not self-consistent, in that the Nonte Carlo bundles do not see each other, and the electric flelds generated between them are ignored. Thls is valid since the density of the high-energy particles is very small compared to the fluld density.

As a demonstration ef this model a code was weltten on a CDC 7600 computer, and was used to calculate the thernonuclear alpha particle hegting of a very dense gas-embedded 7-pinch. ${ }^{6}$ Recent studies have indicated that the $z-p$ inch may have a lifetlme in excess of the shorcest $\ddot{e r o w t h} t$ ime for IHD Instabilicy; ${ }^{7}$ resulting in a renewed interest in the gas-embedded 2 -pinch as a fusion concept. Previous calculations were done on a 2-D MHD code with a crude analytical model to simulate the 


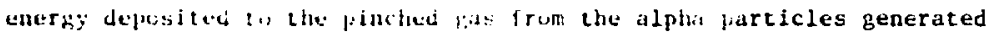
tron thermonacleat reactions. Lik results of this calculution show that the alpha partate a. . Ly depositiuns are in fair agreament with the previous cialculat lons, but that enerby lusses duc to drift are lower than expected. These diferences an be tetributet to the nonlocal energy deposicons of the alpha partide.. dise detajos if this calculation are described in Appendt: 1.

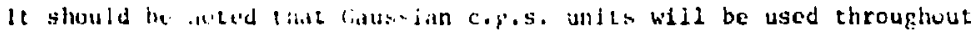
this intuge. 


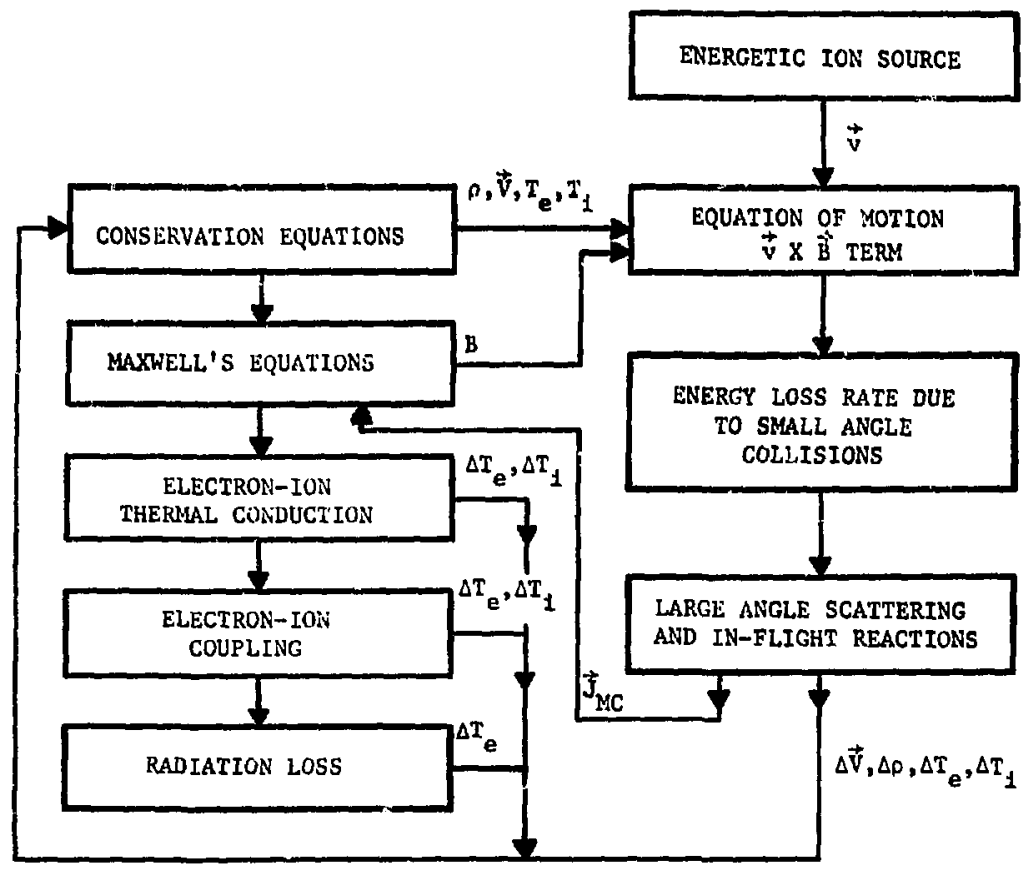

Elgure 1. Block dlegram of MHD-Monte Carlo Model: Left hand part of diagram is the MHD, while that on the right is the Monte Carlo Model. 
2. MHD MODEL

\section{General}

The equations to be solved are:

$$
\begin{aligned}
& \frac{d m}{d t}=\dot{m} \\
& \frac{d \vec{V}}{d t}=-\frac{1}{\rho} \nabla(p+q)+\frac{1}{\rho c} \vec{J} \times \vec{B}+\vec{V}_{M C} \\
& \rho \frac{d \varepsilon_{1}}{d t}=-\rho\left(p_{1}+q\right) \frac{d \tau}{d t}-\omega\left(K T_{1}-K T_{e}\right)-\nabla \cdot \vec{Q}_{1}+s_{i} \\
& \rho \frac{d \varepsilon_{e}}{d t}=-\rho p_{e} \frac{d \tau}{d t}+\omega\left(K T_{1}-K I_{e}\right)-\nabla \cdot \dot{Q}_{e} \\
& +n|\vec{J}|^{2}-p_{B}+s_{G} \\
& \nabla \times \vec{B}=\frac{4 \pi !}{C} \vec{J} \\
& -\frac{1}{c} \frac{d}{d t} \int_{S} \vec{B} \cdot \hat{n} d a=\int_{c} \vec{E} \cdot d \vec{l}
\end{aligned}
$$

with a simple Ohn's law

$$
\overrightarrow{E^{\prime}}=n \vec{j}
$$

$$
\text { where } \quad \begin{aligned}
\rho & =\text { density of fluid } \\
\vec{v} & =\text { velocity of fluid } \\
p_{e} & =\text { electron pressure } \\
n_{1} & =\text { icn nressure } \\
p & =p_{e}+p_{f} \\
q & =\text { artificlial viscosity } \\
n_{e} & =\text { density of electrons }
\end{aligned}
$$




$$
\begin{aligned}
& \mathrm{n}_{i}=\text { densit.y of ions } \\
& \mathrm{m}=\text { mass of fiuid } \\
& n=\text { electric resistivity } \\
& K \quad=\text { Boltzman's constant } \\
& T_{e}=\text { electron temperature } \\
& T_{1}=\text { ion temperature } \\
& \omega=\text { electron-ion coupling coefficient } \\
& {\overrightarrow{q_{1}}}_{1}=\text { lon heat } \mathrm{flux} \text { vector } \\
& \vec{Q}_{\mathrm{z}}=\text { electron heat flux vector } \\
& E_{1}=\text { internal ton energy } \\
& \varepsilon_{\mathrm{e}}=\text { internal electron energy } \\
& \tau \text { = the specific volume (reciprocal of density) } \\
& \dot{m}_{M C}=\text { Nonte Carlo sources to mass } \\
& \dot{\vec{v}}_{\mathrm{MC}}=\text { Monte Carlo sources to momentum } \\
& S_{i}=\text { Nonte Carlo sources to ion energy } \\
& \mathrm{S}_{\mathrm{e}}=\text { Monte Carlo sources to electron energy } \\
& P_{B}=\text { bremsstrahlung energy density loss rate } \\
& \text { है' I electric } \vec{i} \text { 'ield in the rest frame of } d \vec{l}
\end{aligned}
$$

Note that the equations are written in terms of the total derivative which is the form that is differenced in Lagranglan coordinates.

$$
\begin{aligned}
& \text { Since only } \vec{B}=B_{\theta}(R, Z) \hat{\theta} \text { is being consldered } \\
& \vec{Q}_{1}=-\kappa_{\perp 1} \nabla\left(K T_{1}\right) \\
& \vec{Q}_{e}=-\kappa_{\perp e} \nabla\left(K T_{e}\right)
\end{aligned}
$$


and from Glasstone and Lovberg 8

$$
P_{B}=g \frac{32 \pi}{3^{3 / 2}} \cdot \frac{\left(2 \pi K T_{e}\right)^{1 / 2} e^{6}}{m_{e}^{3 / 2} c^{3} h} n_{e} \sum_{i}\left(n_{1} z_{i}^{2}\right)
$$

or

$$
P_{B}=5.35 \times 10^{-24} n_{e} \sum_{i}\left(n_{1} z_{j}^{2}\right) T_{e}^{1 / z} \operatorname{ergs} /\left(\mathrm{cm}^{3} \mathrm{sec}\right)
$$

where $T_{e}$ is given in keV.

From Braginskt. (PB. $217-1.4 \cdot 2.17)^{4}$

$$
w=\frac{3 m_{e}}{m_{1}} \frac{n_{e}}{\tau_{e}} ; \tau_{e}=\frac{3 \sqrt{m_{e}}\left(k T_{e}\right)^{3 / 2}}{4 \sqrt{2 \pi} e^{4} z^{2} n_{1} \ln \Lambda}
$$

where in $\Lambda$ is given by the expression ${ }^{9}$

$$
\ln \Lambda=31.17+\frac{1}{2} \ln \left[\frac{\left(\mathrm{KT}_{\mathrm{e}}\right)^{2}}{\pi_{\mathrm{e}}}\right]
$$

and where $\left(K T_{e}\right)$ is expressed in keV.

The transport coefficlents $\kappa_{1}$, $k_{11}$, and $\sigma_{1}$ were modeled after Braginskil, and are described in Appendix 2.

In addition to these abuve equations, two equations of state are needed. A perfect gas is assumed

$$
\begin{aligned}
& p_{e}=n_{e} K I_{e} \\
& p_{1}=n_{1} K T_{1}
\end{aligned}
$$

Now to solve the above energy equations, a "splitting" technique was used; i.e., the conduction and the joule heating terms were calculated separately and the energy of the zones was adjusted accordingly. 


\subsection{Conservation Equations}

Since the source term $\dot{\mathrm{m}}_{\mathrm{MC}}$ in Eq. (2.0.1) is to be calculated and added to the problem mass independently, Eq. $(2,0.1)$ becomes

$$
\frac{d m}{d t}=0
$$

or the mass in a fluld elemert remains constant. Therefore this equation need not be considered.

Eliminating the current $\vec{J}$ from Eq. (2.0.2) using Eq. (2.0.5) glves:

$$
\frac{d \vec{V}}{d t}=\frac{1}{\rho}\left\{-\nabla p+q-\frac{B_{\theta}}{4 \pi R} \nabla\left(R B_{\theta}\right)\right\}+\vec{V}_{M C}
$$

and splitting off the thermal conduction and joule heating gives for the energy equations

$$
\begin{aligned}
& \rho \frac{d E_{1}}{d t}=-\rho\left(P_{i}+q\right) \frac{d \tau}{d t}-\omega\left(K T_{i}-K T_{e}\right)+s_{1} \\
& \rho \frac{d E_{e}}{d t}=-\rho P_{e} \frac{d \tau}{d t}+\omega\left(K T_{1}-K T_{e}\right)-P_{B}+s_{e}
\end{aligned}
$$

Equations $(2,1.1)-(2.1 .3)$ are now to be differenced for Lagranglan coord1nates, In a manner similar to Schulz. 2,3

Letting $k$ and $\ell$ be defined as Lagrangian coordinates, the Jacoblan of the transformation is given by

$$
j=-\frac{\partial R}{\partial k} \frac{\partial Z}{\partial l}+\frac{\partial R}{\partial l} \frac{\partial Z}{\partial k}
$$




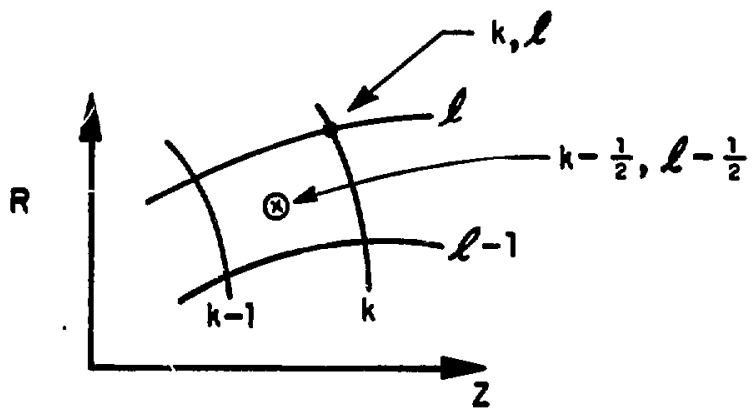

Figure 2. Lagrangian mesh in lilerian reference frame.

Note that $j$ is the negative of the usual expression. To convert Eulerian derivatives to Lagrangial, the following expressions can easily be derived.

$$
\begin{aligned}
& \frac{\partial g}{\partial R}=\frac{1}{J}\left(\frac{\partial Z}{\partial k} \frac{\partial g}{\partial i}-\frac{\partial z}{\partial R} \frac{\partial g}{\partial k}\right) \\
& \frac{\partial g}{\partial z}=\frac{1}{J}\left(\frac{\partial k}{\partial k} \frac{\partial k}{\partial k}-\frac{\partial k}{\partial k} \frac{\partial g}{\partial k}\right)
\end{aligned}
$$

where $h$ is some arbltrary function.

Now consider the two components of the vector Eq. (2.1.1), remembering $\vec{B}=B_{\theta}(R, z) \hat{e}$.

$$
\frac{d V_{R}}{d t}=\frac{1}{q}\left[-\frac{\partial}{\partial R}(p+u)-\frac{B}{4 \pi R} \frac{\partial(R B)}{\partial R}\right]+\left[\ddot{b}_{R}\right]_{N C C}
$$


and

$$
\frac{d v_{7}}{d t}=\frac{1}{\rho}\left[-\frac{\partial}{\partial Z}(p+q)-\frac{B}{4 \pi R} \frac{\partial(R B)}{\partial Z}\right]+\left[\dot{v}_{z}\right]_{M C}
$$

Transforming to Lagrangian coordinates, using (2.1.5) and (2.1.6) gives

$$
\begin{aligned}
& \frac{d V_{R}}{d t}=\frac{i}{\rho J}\left\{\left[\frac{\partial Z}{\partial l} \frac{\partial P}{\partial k}-\frac{\partial Z}{\partial k} \frac{\partial P}{\partial l}\right]\right. \\
&\left.+\frac{B}{4 \pi R}\left[\frac{\partial Z}{\partial l} \frac{\partial(R B)}{\partial k}-\frac{\partial Z}{\partial k} \frac{\partial(R B)}{\partial l}\right]\right\}+\left[\dot{V}_{R}\right]_{M C} \\
& \frac{\partial v_{Z}}{\partial t}-\frac{1}{\rho j}\left\{\left[\frac{\partial R}{\partial k} \frac{\partial P}{\partial l}-\frac{\partial R}{\partial l} \frac{\partial P}{\partial k}\right]\right. \\
&\left.+\frac{B}{4 \pi R}\left[\frac{\partial R}{\partial k} \frac{\partial(R B)}{\partial l}-\frac{\partial R}{\partial l} \frac{\partial(R B)}{\partial k}\right]\right\}+\left[\dot{V}_{Z}\right]_{M C}
\end{aligned}
$$

where

$$
\mathbf{P}=\mathbf{p}+\mathbf{q}
$$

Since the energy equation has no spatial derivat1ves, Eqs. (2.1.2) and (2.1.3) are already in proper form. Now Eqs. (2.1.2), (2.1.3), $(2.1 .7)$, and $(2.1 .8)$ must be differenced. 


\subsection{Difference fuations: Momentum}

To simplify the notation, the following are defined.

$$
\frac{\partial}{\partial k}=\Delta ; \frac{\partial}{\partial z}=\delta
$$

Equation $(2.1 .8)$ becomes

$$
\frac{\partial V_{Z}}{\partial t}-\frac{1}{\rho j}[\Delta R(\delta P+C \delta \Psi)-\delta R(\Delta P+C \Delta \Psi)]+\left(\dot{V}_{Z}\right)_{M C}
$$

where $\Psi=\mathrm{RB}$ and $\mathrm{C}=\mathrm{B} / 4 \pi \mathrm{R}$

Since the momentum is to be calculated at the (k, l) interaectione (see Fig, 2)

$$
\begin{aligned}
& \left(\frac{\partial V_{z}}{\partial t}\right)_{k, \ell}=\left\{\frac{1}{\rho j}[\Delta R(\delta P+c \delta \Psi)-\delta R(\Delta P+c \Delta \Psi)]\right\}_{k, \ell} \\
& +\left\{\left(\dot{V}_{Z}\right)_{M C}\right\}_{k, \ell}
\end{aligned}
$$

This 18 differenced as follows

$$
\begin{aligned}
& {\left[\frac{v_{Z}^{n+1}-v_{Z}^{n}}{\Delta t}\right]_{k, \ell}=\left[\frac{\Delta R}{2 p j}(\delta P+C \delta \Psi)\right]_{k+1 / 2, \ell}^{n}} \\
& +\left[\frac{\Delta R}{2 p j}(\delta P+C \delta \Psi)\right]_{k-1 / 2, \ell}^{n}-\left[\frac{\delta R}{2 p j}(\Delta P+C \Delta \Psi)\right]_{k, \ell+1 / 2}^{n} \\
& -\left[\frac{\delta R}{2 \rho j}(\Delta P+C \Delta \Psi)\right]_{k, \ell-1 / 2}^{n}+\left[\left(\dot{V}_{Z}\right)\right]_{k C, l}^{n+1 / 2}
\end{aligned}
$$

where

$$
\begin{aligned}
& (\delta P)_{k+1 / 2, \ell}=P_{k+1 / 2, \ell+1 / 2}-P_{k+1 / 2, \ell-1 / 2} \\
& (\delta P)_{k-1 / 2, \ell}=P_{k-1 / 2, \ell+1 / 2}-P_{k-1 / 2, \ell-1 / 2} \\
& (\Delta P)_{k, \ell+1 / 2}=P_{k+1 / 2, \ell+1 / 2}-P_{k-1 / 2, \ell+1 / 2}
\end{aligned}
$$




$$
\begin{aligned}
& (\Delta)_{k, \ell-1 / 2}=P_{k+1 / 2, \ell-1 / 2}-P_{k-1 / 2, \ell-1 / 2} \\
& \operatorname{Co}_{k+1 / 2, \ell}=C_{k+1 / 2, \ell}\left(\Psi_{k+1 / 2, \ell+1 / 2}-\Psi_{k+1 / 2, \ell-1 / 2}\right) \\
& C_{k+1 / 2, \ell}=\frac{1}{4 \pi}\left(B_{k+1 / 2, \ell+1 / 2}+B_{k+1 / 2, \ell-1 / 2}\right) \\
& /\left(R_{k, \ell}+R_{k+1, \ell}\right)
\end{aligned}
$$

The remaining derivactves of $\psi$ are differenced analogously.

It 1e assumed here that $P, B$, and $\Psi$ is avallable in the zone centers. The tensor nature of $P$, because of the censor definition of $q$, will be discusged later.

The remaining terms are differenced as follows

$$
\begin{aligned}
& \left(\frac{\Delta R}{2 \rho j}\right)_{k+1 / 2, \ell}=\frac{\left(R_{k+1, \ell}-k_{k, l}\right)}{\left[(o j)_{k+1 / 2, \ell+1 / 2}+(\rho j)_{k+1 / 2, \ell-1 / 2}\right]} \\
& \left(\frac{\Delta R}{2 \rho j}\right)_{k-1 / 2, \ell}=\frac{\left(k_{k, \ell}-R_{k-1, \ell}\right)}{\left[(\rho j)_{k-1 / 2, \ell+1 / 2}+(\rho j)_{k-1 / 2, \ell-1 / 2}\right]} \\
& \left(\frac{\delta R}{2 \rho j}\right)_{k, \ell+1 / 2}=\frac{\left(R_{k, \ell+1}-R_{k, l}\right)}{\left[(\rho j)_{k+1 / 2,2+1 / 2}+(\rho j)_{k-1 / 2, \ell+1 / 2}\right]} \\
& \left(\frac{\delta R}{2 \rho j}\right)_{k, \ell-1 / 2}=\frac{\left(R_{k, \ell}-R_{k, \ell-1}\right)}{\left[(\rho j)_{k+1 / 2, \ell-1 / 2}+(\rho j)_{k-1 / 2, \ell-1 / 2}\right]}
\end{aligned}
$$

Note the $(\rho j)$ terms are all zone centered. The density $p$ is zone centered by definition, but now the area Jacobian, $j$, must be calculated In the zone center. 
Repeating Eq. (2.1.4) and using new notation

$$
j=\frac{\partial R}{\partial l} \frac{\partial Z}{\partial k}-\frac{\partial R}{\partial k} \frac{\partial Z}{\partial l}=\delta R \Delta Z-\Delta R \delta Z
$$

and according to Schulz 2,3

$$
j_{k-1 / 2, \ell-1 / 2}=1 / 2\left(j_{k, \ell}+j_{k-1, \ell-1}\right)
$$

where

$$
\begin{aligned}
J_{k, \ell}= & \left(R_{k, \ell}-R_{k, \ell-1}\right)\left(z_{k, \ell}-z_{k-1, \ell}\right) \\
& -\left(R_{k, \ell}-R_{k-1, \ell}\right)\left(z_{k, \ell}-z_{k, \ell-1}\right)
\end{aligned}
$$

and similarly

$$
\begin{gathered}
j_{k-1, \ell-1}=\left(R_{k-1, \ell}-R_{k-1, \ell-1}\right)\left(z_{k, \ell-1}-z_{k-1, \ell-1}\right) \\
-\left(R_{k, \ell-1}-R_{k-1, \ell-1}\right)\left(z_{k-1, \ell}-z_{k-1, \ell-1}\right)
\end{gathered}
$$

It is interesting to note that $f_{k-1 / 2, \ell-1 / 2}$ is the exact area of the quadrilateral (zone) that it is centered in. This can be seen by close inspection of the terms and noting that $j_{k, \ell}$ is the area of a parallelogram defined by its adjacent sides.

The Monte Carlo transport term $\overrightarrow{\dot{v}}_{M C}$ is calculated at time $\mathfrak{n}+1 / 2$, and is zone centered by definition. Therefore $\left[\dot{V}_{Z}\right]_{M C}$ must be averaged.

$$
\begin{aligned}
& {\left[\left(\dot{V}_{Z}\right)_{M C}\right]_{k, \ell}=\left[\left(m \dot{V}_{2}\right)_{k+1 / 2, \ell+1 / 2}^{M C}+\left(m \dot{V}_{Z}\right)_{k+1 / 2, \ell-1 / 2}^{M C}\right.} \\
& \left.+\left(m \dot{v}_{z}\right)_{k-1 / 2, \ell+1 / 2}^{M C}+\left(m \dot{v}_{z}\right)_{k-1 / 2, \ell-1 / 2}^{M C C}\right] /\left[\mathbb{m}_{k+1 / 2, \ell+1 / 2}\right. \\
& \left.+m_{k+1 / 2, \ell-1 / 2}+m_{k-1 / 2, \ell+1 / 2}+m_{k-1 / 2, \ell-1 / 2]}\right]
\end{aligned}
$$


This concludes the differencing of the axial momentum equation.

The radial momentum equation should be differenced in a similar manner

$$
\begin{aligned}
& {\left[\frac{v_{R}^{n+1}-v_{R}^{n}}{\Delta t}\right]_{k, \ell}=\left[\frac{\delta Z}{20} j(\Delta P+c \Delta \psi)\right]_{k, k,-1 / 2}^{n}} \\
& +\left[\frac{\delta Z}{2 \rho J}(\Delta \mathrm{P}+\mathrm{C} \Delta \psi)\right]_{k, \ell+1 / 2}^{\mathrm{n}}-\left[\frac{\delta Z}{2 \rho j}(\delta \mathrm{P}+\mathrm{c} \delta \psi)\right]_{k+1, \ell}^{\mathrm{n}} \\
& -\left[\frac{\Delta Z}{2 p J}(\delta P+C \delta \Psi)\right]_{k-1 / 2, \varepsilon}^{n}+\left[\left(\dot{V}_{R}\right){ }_{N C}\right]_{k, \ell}^{n+1 / 2}
\end{aligned}
$$

Note that $(\rho \mathrm{J}), \delta P, \Delta P, C \delta \psi, C \Delta \psi$ have all prevlously been defined in Eqs. (2.2.1a-f) for this $s t a t$ of $k$ and $\ell$, therefore the remaining terms are def ined as :

$$
\begin{aligned}
& (\delta z)_{k, \ell-1 / 2}=z_{k, \ell}-z_{k, \ell-1} \\
& (\delta 2)_{k, \ell+1 / 2}=z_{k, \ell+1}-z_{k, \ell} \\
& (\Delta z)_{k+1 / 2, \ell}=z_{k+1, \ell}-z_{k, \ell} \\
& (\Delta z)_{k-1 / 2, \ell}=z_{k, \ell}-z_{k-1, \ell}
\end{aligned}
$$

The Monte Carlo transport term, $\dot{V}_{R}$, is averaged in a manner Identical to Eq. $(2,2,5)$.

The above Eqs. (2.2.1) and (2.2.6) are the Lagrangian difference equations for momentuni conservation in cylindrical coordinates. The only remaining loose end is tine calculation of the $P$ terms 


$$
\begin{aligned}
& P \equiv p+q \\
& P_{k-1 / 2, \ell-1 / 2}=p_{k-1 / 2, \ell-1 / 2}+q_{k-1 / 2, \ell-1 / 2}^{1}
\end{aligned}
$$

where

$$
p_{k-1 / 2, \ell-1 / 2}=\left[n_{e} K T_{e}+n_{i} K T_{i}\right]_{k-1 / 2, \ell-1 / 2}
$$

and $q^{1}$ syubolizes the tensor nature of $q$. Four $q$ terms $w 111$ be defined; cach a measure of the compression alang the four sides of the quadrilateral $(k-1 / 2, l-1 / 2)$. Let $q^{1}$ be assoclated with side $(k-1 / 2,2)$, and $q^{2}, q^{3}, q^{4}$ for the remaining sides going clockwise arnund the zone. (See Fig. 3.)

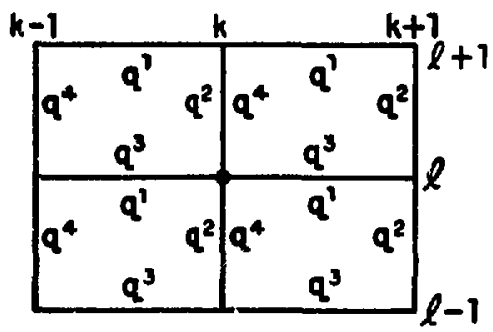

F1gure 3. Quadrilateral zones with tensor artificial viscosities. 
The actual deitintion of $q$ is discussed in Appendix 3 . The four $q^{1}$ terms can now be inserted into Eqs. $(2.2 .1 a)-(2.2 .1 d)$. For example:

$$
\begin{aligned}
(\delta P)_{k+1 / 2, \ell}= & {\left[\delta\left(p+q^{4}\right)\right]_{k+1 / 2, \ell} } \\
= & p_{k+1 / 2, l+1 / 2}+q_{k+1 / 2, \ell+1 / 2}^{4}-p_{k+1 / 2, \ell-1 / 2} \\
& -q_{k+1 / 2, \ell-1 / 2}^{4}
\end{aligned}
$$

Note that $(\delta P)_{k+1 / 2, l}$ is the pressure gradient with respect to $l$, $\delta \equiv \frac{\partial}{\partial l}$, and to the right of the point $k, l$. Therefore the correct $q^{i}$ to use is $q^{4}$, similarly for the others

$$
\begin{aligned}
& (\delta P)_{k-1 / 2, \ell} *\left[\delta\left(p+q^{2}\right)\right]_{k-1 / 2, l} \\
& (\Delta P)_{k, \ell+1 / 2} \approx\left[\Delta\left(p+q^{3}\right)\right]_{k, \ell+1 / 2} \\
& (\Delta P)_{k, \ell-1 / 2}=\left[\Delta\left(p+q^{3}\right)\right]_{k, \ell-1 / 2}
\end{aligned}
$$

Equations $(2.2 .1)$ and $(2.2 .6)$ are now eas1ly solved for $v_{z}^{n+1}$ and $v_{R}^{n+k}$ for a given $\Delta t$. After solving for $v_{Z}^{n+1}$ and $v_{R}^{n+1}$, Integrating again gives

$$
\begin{aligned}
& R^{n+1}=R^{n}+v_{R}^{n+1} \Delta c \\
& z^{n+1}=z^{n}+v_{z}^{n+1} \Delta t
\end{aligned}
$$

This concludes the discussion of the momentum equations and now the energy equations will be discussed. 


\subsection{Difference Equacions: Energy}

The energy equations to be differenced are Eqs. (2.1.2) and (2.1.3). Remember that the thermal conduction and joule leating have been split from the equation and their contributions to the energy transport will be calculated separately. The electron and fon equations are differenced in time as follow:

$$
\begin{aligned}
& \varepsilon_{1}^{n+1}-\varepsilon_{i}^{n}+\left(\frac{p_{1}^{n+1}}{2}+\frac{p_{1}^{n}}{2}+q^{n+1 / 2}\right) d \tau^{n+1 / 2} \\
& +(\omega \tau)^{n+1}\left(k \tau_{1}^{n+1}-k 1_{e}^{n+1}\right) \Delta t-\left(S_{1} \tau\right)^{n+1 / 2} \Delta t=0 \quad(2.3 .1) \\
& \varepsilon_{e}^{n+1}-\varepsilon_{e}^{n}+\left(\frac{p_{c}^{n+1}}{2}+\frac{p_{e}^{n}}{2}\right) d_{\tau}^{n+1 / 2}-(\omega \tau)^{n+1}\left(K \tau_{1}^{n+1}-K T_{e}^{n+1}\right) \Delta t \\
& \quad+\left(p_{B}^{\tau}\right)^{n+1 / 2} \Delta t-\left(s_{e} \tau\right)^{n+1 / 2} \Delta t=0
\end{aligned}
$$

Now let

$$
\begin{aligned}
& r_{i}=n_{i} k T_{1} \\
& p_{e}=n_{e} k T_{e} \\
& \varepsilon_{1}=\frac{3}{2} n_{i} k_{i} \\
& \varepsilon_{e}=\frac{3}{2} n_{e} k_{k T}
\end{aligned}
$$

and substitute these into the above equations and solve for $k T_{1}^{n+1}$ and $\mathrm{kI}_{\mathrm{e}}^{\mathrm{n+1}}$. After a 11ttle algebra, Eqs. (2.3.1) and (2.3.2) become

$$
K T_{i}^{n+1}=\frac{B}{A}+\frac{C}{A} K T_{e}^{n+1}
$$


where $A, B$, and $C$ are defined as follows

$$
\begin{aligned}
A= & \frac{3}{2}\left(n_{i} \tau\right)^{n+1}+\frac{1}{2} n_{i}^{n+1} d \tau^{n+1 / 2}+(\omega \tau)^{n+1} \Delta t \\
B= & \frac{3}{2}\left(n_{i} \tau\right)^{n} K T_{1}^{n}-\left(\frac{1}{2} n_{i}^{n} k T_{1}^{n}+q^{n+1 / 2}\right) d \tau^{n+1 / 2} \\
& +\left(S_{i} \tau\right)^{n+1 / 2} \Delta t \\
C= & (\omega \tau)^{n+1} \Delta t
\end{aligned}
$$

similarly for the electron equation

$$
K T_{e}^{n+1}-\frac{E}{D}+\frac{E}{D} K T_{1}^{n+1}
$$

where

$$
\begin{aligned}
D= & \frac{3}{2}\left(n_{e} \tau\right)^{n+1}+\frac{1}{2} n_{e}^{n+1} d \tau^{n+1 / 2}+(\omega \tau)^{n+1} \\
E= & \frac{3}{2}\left(n_{e} \tau\right)^{n} k T_{e}^{n}-\frac{1}{2} n_{e}^{n} k T_{e}^{n} d \tau^{n+1 / 2}-\left(P_{B} \tau\right)^{n+1 / 2} \Delta t \\
& +\left(S_{e} \tau\right)^{n+1 / 2} \Delta t \\
F= & (\omega \tau)^{n+1} \Delta t
\end{aligned}
$$

Now use Eq. $(2.3 .4)$ to solve $(2.3 .3)$

$$
K T_{1}^{n+1} \approx \frac{D B+C F}{A D-C F}
$$

Now $\mathrm{KT}_{1}^{\mathrm{n}+1}$ can be used to calculate $\mathrm{KI}_{\mathrm{e}}^{\mathrm{n}+1}$ from Eq. (2.3.4). This completes the time differencing and now the spatlal averaging will be discussed.

We want the energy to be a zone centered quant1ty, and al1 the variables, except $q$, are zone centered, so the rest is simple. For a scalar, zone centered q, which is needed in Eq. $(2.3,3 b)$, use 


$$
q_{k-1 / 2, \ell-1 / 2}^{n+1 / 2}=\frac{1}{2}\left(q^{n+1}+q^{n}\right)_{k-1 / 2, \ell-1 / 2}
$$

and let

$$
q_{k-1 / 2, \ell-1 / 2}=\frac{1}{2}\left(q^{1}+q^{2}+q^{3}+q^{4}\right)_{k-1 / 2, \ell-1 / 2}
$$

Note that $q^{n+1}$ is calculated after the momentum, when the coordinates and velocities are known at time $n+1$. Likewise

$$
\begin{aligned}
& d \tau^{n+1 / 2}=\tau^{n+1}-\tau^{n} \\
& \tau^{n+1 / 2}=\frac{1}{2}\left(\tau^{n+1}+\tau^{n}\right)
\end{aligned}
$$

where $\tau^{n+1}$ can be calculated after the momentum

$$
\begin{aligned}
\tau_{k-1 / 2, \ell-1 / 2}^{n+1} & =\frac{1}{m_{k-1 / 2, \ell-1 / 2}}\left\{\frac { \pi } { 3 } \left[j _ { k , \ell } ^ { n + 1 } \left(R_{k-1, \ell}^{n+1}+R_{k, \ell-1}^{n+1}\right.\right.\right. \\
\left.+k_{k, \ell}^{n+1}\right) & \left.\left.+j_{k-1, \ell-1}^{n+1}\left(R_{k-1, \ell}^{n+1}+R_{k, \ell-1}^{n+1}+R_{k-1, \ell-1}^{n+1}\right)\right]\right\}
\end{aligned}
$$

The terni in the curled brackets $\{\ldots\}$ is the volume of the zone, and the $f$ terms are as defined in the previous section on momentum Eqs. $(2,2,3)$ and $(2,2,4)$.

Except for joule heating and thermal conduction, chis completes the section on differencing the energy equation. Since the joule heating and thermal conduction depend on solving the magnetic and thermal diffusion equations, it is more convenient to describe the solutions to these equations first end then update the energy equations. 


\subsection{Magnetic Field Diffusion}

The time and space variations of the magnetic field were solved by casting the field equations into the form of a diffusion equation. This diffusion equation was then solved implicitly by the splitting method. Using Eq. (2.0.7) in Eq. (2.0.6) gives

$$
-\frac{1}{c} \frac{d}{d t} \int_{s} \vec{B} \cdot \hat{n} d a=\int n \vec{J} \cdot d \vec{l}
$$

Use Stokes theorein:

$$
-\frac{1}{c} \frac{d}{d t} \int_{s} \vec{B} \cdot \hat{n} d a=\int_{s} \nabla x(n \vec{J}) \cdot \hat{n} d a
$$

and now use Eq. (2.0.5) to eliminate $ఫ$

$$
-\frac{1}{c} \frac{d}{d t} \int_{S} \vec{B} \cdot \hat{n} d a=\int_{S} \nabla \times\left[n \frac{c}{4 \pi} \nabla \times \vec{b}\right] \cdot \hat{n} d a
$$

or

$$
\frac{1}{A} \frac{d \Phi}{d t}=-\frac{c^{2}}{4 \pi} \nabla \times(n \nabla \times \vec{B}) \cdot \hat{n}
$$

where $\Lambda=$ area ( $A$ will equal the zonal area, and $\vec{B}$ is parallel to the normal of $A$ ) and $\Phi=B A$ is the magnetic flux

Letting $\vec{B}=\frac{\Psi}{R} \hat{\theta}$ and expanding the cross products gives

$$
\frac{1}{A} \frac{d \Phi}{d t}=\frac{c^{2} R}{l \pi} \nabla \cdot\left(\frac{n}{R^{2}} \nabla \psi\right)
$$

This is a diffusion equation, that can now be differenced implicitly. Transform this equation to Lagranglan form, using the transform Egs. $(2.1 .5)$ and $(2.1 .6)$ 


$$
\begin{aligned}
& \frac{1}{A} \frac{d s}{d t}=\frac{c^{2} R}{4 \pi} \nabla \cdot \hat{i} \frac{\eta}{R^{2} j}\left(-\frac{\partial 2}{\partial l} \frac{\partial \psi}{\partial k}+\frac{\partial \ddot{t}}{\partial k} \frac{\partial \psi}{\partial l}\right) \\
& +\hat{k} \frac{n}{k^{2} j}\left(\frac{\partial R}{\partial l} \frac{\partial \psi}{\partial k}-\frac{\partial R}{\partial k} \frac{\partial \psi}{\partial l}\right) \hat{l}
\end{aligned}
$$

Fixpanding the divergence for cylindrical coordinates gives

$$
\begin{aligned}
\frac{1}{A} \frac{d \phi}{d t} & =\frac{c^{2} R}{4 \pi}\left\{\frac{1}{R j}\left[-\frac{\partial Z}{\partial l} \frac{\partial}{\partial k}+\frac{\partial Z}{\partial k} \frac{\partial}{\partial l}\right] \frac{n}{R j}\left[-\frac{\partial Z}{\partial l} \frac{\partial \psi}{\partial k}+\frac{\partial Z}{\partial k} \frac{\partial \psi}{\partial \ell}\right]\right. \\
& \left.+\frac{1}{j}\left[\frac{\partial R}{\partial l} \frac{\partial}{\partial k}-\frac{\partial R}{\partial k} \frac{\partial}{\partial l}\right] \frac{n}{R^{2} j}\left[\frac{\partial R}{\partial l} \frac{\partial \psi}{\partial k}-\frac{\partial R}{\partial k} \frac{\partial \psi}{\partial l}\right]\right\}
\end{aligned}
$$

Aiter some algebra this reduces to

$$
\begin{aligned}
\frac{1}{A} \frac{\partial \phi}{\partial t} & =\frac{c^{2}}{4 \pi j}\left\{\frac{\partial}{\partial k}\left[\frac{\eta\left(\frac{\partial Z}{\partial l}\right)^{2}}{R j} \frac{\partial \psi}{\partial k}\right]-\frac{\partial}{\partial k}\left[\frac{\eta\left(\frac{\partial z}{\partial l}\right)\left(\frac{\partial z}{\partial k}\right)}{R j} \frac{\partial \psi}{\partial l}\right]\right. \\
& -\frac{\partial}{\partial l}\left[\frac{\eta\left(\frac{\partial z}{\partial k}\right)\left(\frac{\partial z}{\partial l}\right)}{R j} \frac{\partial \psi}{\partial k}\right]+\frac{\partial}{\partial l}\left[\frac{\eta\left(\frac{\partial z}{\partial k}\right)^{2}}{R j} \frac{\partial \psi}{\partial l}\right] \\
& +\frac{\partial}{\partial k}\left[\frac{\eta\left(\frac{\partial R}{\partial l}\right)^{2}}{R j} \frac{\partial \psi}{\partial k}\right]-\frac{\partial}{\partial k}\left[\frac{n\left(\frac{\partial R}{\partial l}\right)\left(\frac{\partial R}{\partial k}\right)}{R j} \frac{\partial \psi}{\partial l}\right] \\
& \left.\left.-\frac{i}{\partial l}\left[\frac{n\left(\frac{\partial R}{\partial k}\right)\left(\frac{\partial k}{\partial l}\right)}{R j}\right] \frac{\partial \psi}{\partial k}\right]+\frac{\partial}{\partial l}\left[\frac{n\left(\frac{\partial R}{\partial k}\right)^{2}}{R j} \frac{\partial \psi}{\partial l}\right]\right\}
\end{aligned}
$$

Lit $\quad \frac{\partial R}{\partial R}=R_{\ell}, \frac{\partial R}{\partial k}=R_{k}$, etc.

Making the approximation that the cross terms are smal1 10,11 compared to the others the above becomes

$$
\frac{1}{\Lambda} \frac{\partial \Phi}{\partial t}=\frac{c^{2}}{4 \pi j}\left\{\frac{\partial}{\partial k} \frac{n\left(R_{k}^{2}+z_{l}^{2}\right)}{R j} \frac{\partial \psi}{\partial k}+\frac{\partial}{\partial \ell} \frac{\eta\left(R_{k}^{2}+z_{k}^{2}\right)}{R J} \frac{\partial \psi}{\partial \ell}\right\}
$$

It is this equation which will be diffeienced. Since the magnetic field is to be defined at the zone center, Eq. (2.4.1) should be calculated at $(k-1 / 2, \&-1 / 2)$. Conslder the terms separately. 
The term $\left(\frac{c^{2}}{4 \pi j}\right)_{k-1 / 2, \ell-1 / 2}$ is easily obtained using Eq, $(2,2,3)$, $(2.2 .4),(2.2 .2)$.

The terins in the brackets become

$$
\begin{aligned}
& {\left[\frac{\partial}{\partial k} \frac{n\left(R_{l}^{2}+z_{l}^{2}\right)}{R j} \frac{\partial \psi}{\partial k}\right]_{k-1 / 2, \ell-1 / 2}=\left[\frac{n\left(R_{l}^{2}+z_{l}^{2}\right)}{R j} \frac{\partial \psi}{\partial k}\right]_{k, \ell-1 / 2}} \\
& -\left[\frac{n\left(R_{l}^{2}+z_{l}^{2}\right)}{R j} \frac{\partial \psi}{\partial k}\right]_{k-1, \ell-1 / 2} \\
& {\left[\frac{\partial}{\partial l} \frac{n\left(R_{k}^{2}+z_{k}^{2}\right)}{R_{j}^{j}} \frac{\partial \psi}{\partial \ell}\right]_{k-1 / 2, \ell-1 / 2}=\left[\frac{n\left(R_{k}^{2}+z_{k}^{2}\right)}{R j} \frac{\partial \psi}{\partial \ell}\right]_{k-1 / 2, \ell}} \\
& -\left[\frac{n\left(R_{k}^{2}+z_{k}^{2}\right)}{R j} \frac{\partial \psi}{\partial l}\right]_{k-1 / 2, \ell-1}
\end{aligned}
$$

Now difference the remaining derivatives of $\downarrow$ and rearrange terms

$$
\begin{aligned}
{\left[\frac{1}{\beta A} \frac{\partial \phi}{\partial t}\right]_{k-1 / 2, \ell-1 / 2}=\xi_{1}\left(\psi_{k+1 / 2, \ell-1 / 2}-\psi_{k-1 / 2, \ell-1 / 2}\right) } \\
\quad-\xi_{2}\left(\psi_{k-1 / 2, \ell-1 / 2}-\psi_{k-3 / 2, \ell-1 / 2}\right) \\
\quad+\xi_{3}\left(\psi_{k-1 / 2, \ell+1 / 2}-\psi_{k-1 / 2, \ell-1 / 2}\right) \\
\quad-\xi_{4}\left(\psi_{k-1 / 2, \ell-1 / 2}-\psi_{k-1 / 2, \ell-3 / 2}\right)
\end{aligned}
$$

where

$$
\begin{aligned}
& \xi_{1}=\left[\frac{n\left(R_{\ell}^{2}+z_{l}^{2}\right)}{R j}\right]_{k, \ell-1 / 2} \\
& \xi_{2}=\left[\frac{n\left(R_{l}^{2}+z_{\ell}^{2}\right)}{R_{j}}\right]_{k-1, \ell-1 / 2}
\end{aligned}
$$




$$
\begin{aligned}
& \zeta_{3}=\left[\frac{n\left(k_{k}^{2}+z_{k}^{2}\right)}{k J}\right]_{k-1 / 2,3} \\
& \zeta_{4}=\left[\frac{n\left(k_{k}^{2}+z_{k}^{2}\right)}{k j}\right]_{k-1 / 2, i-1}
\end{aligned}
$$

where

$$
\therefore=\left(\frac{c^{2}}{4 \pi j}\right)_{k-1 / 2,0-1 / 2}
$$

Note that $c_{1}-t_{4}$ are def lued at the zone boundarles, therefore tiey wete iveraged ill the following: wily

$$
\begin{aligned}
& c_{1}=\frac{4 \pi\left[u_{k+1 / 2,1,-1 / 2}+\left(k_{k-1 / 2, k-1 / 2}\right)\right.}{\left(\frac{V}{n}\right)_{k+1 / 2, i-1 / 2}+\left(\frac{V}{n}\right)_{k-1 / 2, k-1 / 2}} \\
& \varepsilon_{2}=\frac{4 \pi\left[a_{k-1 / 2, k-1 / 2}+a_{k-3 / 2, l-1 / 2}\right]}{\left(\frac{V}{\eta}\right)_{k-1 / 2, k-1 / 2}+\left(\frac{v}{\pi}\right)_{k-3 / 2, i-1 / 2}} \\
& E_{3}=\frac{4 \pi\left[\gamma_{k-1 / 2, i+1 / 2}+\gamma_{k-1 / 2, i-1 / 2}\right]}{\left(\frac{V}{n}\right)_{k-1 / 2, i+1 / 2}+\left(\frac{V}{n}\right)_{k-1 / 2, i-1 / 2}} \\
& \xi_{4}=\frac{4 \pi\left(r_{k-1 / 2, l-1 / 2}+\gamma_{k-1 / 2, k-3 / 2}\right)}{\left(\frac{v}{n}\right)_{k-1 / 2, \ell-1 / 2}+\left(\frac{v}{n}\right)_{k-1 / 2, f-3 / 2}}
\end{aligned}
$$

where

$$
\begin{aligned}
& a=\left(R_{k}^{2}+z_{k}^{2}\right) \\
& y=\left(R_{k}^{2}+z_{k}^{2}\right) \\
& V=\text { volume of zone } \cong 2 \pi R J
\end{aligned}
$$


and

$$
\begin{aligned}
& R_{\ell}=\frac{1}{2}\left[\left(R_{k, \ell}-R_{k, \ell-1}\right)+\left(R_{k-1, \ell}-R_{k-1, \ell-1}\right)\right] \\
& z_{\ell}=\frac{1}{2}\left[\left(z_{k, \ell}-z_{k, \ell-1}\right)+\left(z_{k-1, \ell}-z_{k-1, \ell-1}\right)\right] \\
& R_{k}=\frac{1}{2}\left[\left(R_{k, \ell}-R_{k-1, \ell}\right)+\left(R_{k, \ell-1}-B_{k-1, \ell-1}\right)\right] \\
& z_{k}=\frac{1}{2}\left[\left(z_{k, \ell}-z_{k-1, \ell}\right)+\left(z_{k, \ell-1}-z_{k-1, \ell-1}\right)\right]
\end{aligned}
$$

The volume $V$ is calculated as described in Eq. (2.3.6). Also note that $n$, the electric resistivity is defined in the zone center. Thus using the above definitions, Eq. (2.4.2) has been spatially differenced. The magnetic diffusion must now be differenced with respect to t1me.

Noting that $\xi_{1}$ and $\xi_{2}$ are differences in $k$ and $\xi_{3}$ and $\xi_{4}$ are differences in $\ell$, an implicit splitcing scheme may be trled.

$$
\begin{aligned}
\phi^{n^{\prime}} & =A B(\Delta t)\left[\xi_{3} \psi_{k-1 / 2, \ell+1 / 2}^{n^{\prime}}-\xi_{3} \psi_{k-1 / 2, \ell-1 / 2}^{n^{\prime}}\right. \\
& \left.-\xi_{4} \psi_{k-1 / 2, \ell-1 / 2}^{n^{\prime}}+\xi_{4} \psi_{k-1 / 2, \ell-3 / 2}^{n^{\prime}}\right]+\phi^{n} \\
\phi^{n+1} & =A B(\Delta t)\left[\xi_{1} \psi_{k+1 / 2, \ell-1 / 2}^{n+1}-\xi_{1} \psi_{k-1 / 2, \ell-1 / 2}^{n+1}\right. \\
& \left.-\xi_{2} \psi_{k-1 / 2, \ell-1 / 2}^{n+1}+\xi_{2} \psi_{k-3 / 2, \ell-1 / 2}^{n+1}\right]+\phi^{n^{\prime}}
\end{aligned}
$$

noting that $j=A, B=\frac{c^{2}}{4 \pi j}$ and $\phi=\frac{A}{R} \psi$ and after a little algebra the above two equations can be written in the tridiagonal form

$$
\begin{aligned}
& \text { - a } \psi_{k-1 / 2, \ell+1 / 2}^{n^{\prime}}+b \psi_{k-1 / 2, \ell-1 / 2}^{n^{\prime}}-c \psi_{k-1 / 2, \ell-3 / 2}^{n^{\prime}}=d \\
& \text { - e } \psi_{k+1 / 2, \ell-1 / 2}^{n+1}+f \psi_{k-1 / 2, \ell-1 / 2}^{n+1}-g \psi_{k-3 / 2, \ell-1 / 2}^{n+1}=h
\end{aligned}
$$


where

$$
\begin{aligned}
& a=\frac{c^{2} \Delta t}{4 \pi} \xi_{3} \\
& b=\left[\left(\frac{\Lambda}{R}\right)^{n+1}+\frac{c^{2} \Delta t}{4 \pi}\left(\xi_{3}+\xi_{4}\right)\right] \\
& c=\frac{c^{2} \Delta t}{4 \pi} \quad \varepsilon_{4} \\
& d=\left(\frac{A}{R}\right)^{n} \psi_{k-1 / 2, k-1 / 2}^{n} \\
& e=\frac{c^{2} \Delta t}{4 \pi} \xi_{1} \\
& f=\left[\left(\frac{A}{R}\right)^{n+1}+\frac{c^{2} \Delta t}{4 \pi}\left(\xi_{1}+\xi_{2}\right)\right] \\
& g=\frac{c^{2} \Delta t}{4 \pi} \xi_{2} \\
& h=\left(\frac{A}{R}\right)^{n^{\prime}} \psi_{k-1 / 2, \ell-1 / 2}^{n^{\prime}}
\end{aligned}
$$

It is assumed that the coeffictents $a-h$ are centered at $(k-1 / 2$, 2-1/2). This is slightly in error since $\xi_{1}-\xi_{4}$ are 1 in the centers of a zone boundary. It is to be noted that time n' is equal to time $n+1$. Equations $(2.4 .3)$ and $(2.4 .4)$ are now easily solved using the standard tridlagonal technique.

$$
\begin{aligned}
& \psi_{k-1 / 2, \ell-1 / 2}^{n^{\prime}}=E_{k-1 / 2, \ell-1 / 2}^{n^{\prime}} \psi_{k-1 / 2, \ell+1 / 2}^{n^{\prime}}+F_{k-1 / 2, \ell-1 / 2}^{n^{\prime}} \\
& E_{k-1 / 2, \ell+1 / 2}^{n^{\prime}}=\frac{a_{k-1 / 2, \ell+1 / 2}}{b_{k-1 / 2, \ell+1 / 2}-c_{k-1 / 2, \ell+1 / 2} E_{k-1 / 2, \ell-1 / 2}^{n^{\prime}}}
\end{aligned}
$$




$$
\begin{aligned}
& F_{k-1 / 2, \ell+1 / 2}^{n^{\prime}}=\frac{c_{k-1 / 2, \ell+1 / 2} F_{k-1 / 2, \ell-1 / 2}^{n^{\prime}}+d_{k-1 / 2, k+1 / 2}}{b_{k-1 / 2, \ell+1 / 2}-c_{k-1 / 2, i+1 / 2} k_{k-1 / 2, i-1 / 2}^{n^{\prime}}} \\
& \psi_{k-1 / 2,2-1 / 2}^{n+1}=c_{k-1 / 2, \ell-1 / 2}^{n+1} \psi_{k+1 / 2, \ell-1 / 2}^{n+1}+H_{k-1 / 2, \ell-1 / 2}^{n+1} \\
& G_{k+1 / 2, \ell-1 / 2}^{n+1}-\frac{e_{k+1 / i_{2} \ell-1 / 2}}{f_{k+1 / 2, \ell-1 / 2}-g_{k+1 / 2, \ell-1 / 2} G_{k-1 / 2, \ell-1 / 2}^{n+1}} \\
& H_{k+1 / 2, \ell-1 / 2}^{n+1}=\frac{8_{k+1 / 2, \ell-1 / 2} 4_{k-1 / 2, l-1 / 2}^{n+1}+h_{k+1 / 2, \ell-1 / 2}}{f_{k+1 / 2, \ell-1 / 2}-8_{k+1 / 2, \ell-1 / 2} G_{k-1 / 2, \ell-1 / 2}^{n+1}}
\end{aligned}
$$

Computationally, dumy zones are carried along the boundaries, where boundary values of $\psi$ can be stored.

Using the sbove methods, the values of $\psi$ at time $n+1$ are calculated, and since $\psi=B R$, B at $n+1$ is known. 


\subsection{Joule Heating}

Having described the magnetic diffusion, It is convenient now to derive the joule heating term. Since the energy equation has already been differenced without joule heating, it is only necessary to calculate the incremental change to the zone's energy due to the joule hating. The heating will he added to the electrons. Let $E_{e}^{J}$ denote the energy deposited due to joule heat Ing. For a simple Ohm's law,

$$
E_{e}^{J}=\iint d v d t n|\vec{J}|^{2}
$$

and for a particular zone of volume $V$

$$
\Delta E_{e}^{J}=v(\Delta t) n|\vec{n}|^{2}
$$

$$
\text { Now let } \vec{J}=\frac{C}{4 \pi} \nabla \times \vec{B}, \vec{B}=B_{0}(k, z) \hat{\theta} \text {, and } \psi=B_{\theta} R \text {. }
$$

Equation (2.5.1) becomes

$$
\Delta E_{e}^{J}=v(\Delta t) n\left(\frac{c}{4 \pi R}\right)^{2}\left[\left(\frac{\partial \psi}{\partial Z}\right)^{2}+\left(\frac{\partial \psi}{\partial R}\right)^{2}\right]
$$

This equation now has to be transformed to Lagranglan coordinates. Lsjug Eqs. (2.1.5) and (2.1.6), expanding, and dropping the cross terms with the assumption they are small compared to the others, gives the following

$$
\begin{aligned}
& \Delta \mathrm{E}_{\mathrm{e}}^{\mathrm{J}}=\mathrm{V}(\Delta \mathrm{t})\left(\frac{\mathrm{c}}{4 \pi}\right)^{2}\left[\frac{\mathrm{n} \cdot \mathrm{g}^{2} \mathrm{j}^{2}}{\mathrm{f}^{2}}\left(\mathrm{R}_{\mathrm{k}}^{2}+\mathrm{z}_{\mathrm{k}}^{2}\right)\left(\frac{\partial \psi}{\partial l}\right)^{2}\right. \\
& \left.\left.+\left(R_{l}^{2}+z_{\ell}^{2}\right)\left(\frac{\partial \psi}{\partial k}\right)^{2}\right\}\right]
\end{aligned}
$$

The volume of a Lagrangian zone can be approximated by

$$
V=2 \pi j<R>
$$


where $\langle R\rangle$ is the average $R$ of the zone, i.e.,

$$
\langle R\rangle=R_{k-1 / 2, i-1 / 2}
$$

Therefore

$$
\Delta \mathrm{E}_{\mathrm{e}}^{\mathrm{J}}=\frac{c^{2}(\Delta t)}{8 \pi}\left(\frac{\eta}{\nabla_{j}}\right)\left[\left(\mathrm{R}_{k}^{2}+z_{k}^{2}\right)\left(\frac{\partial \psi}{\partial \varepsilon_{i}}\right)^{2}+\left(k_{l}^{2}+z_{l}^{2}\right)\left(\frac{\partial \psi_{l}}{\partial k}\right)^{2}\right]
$$

Ey uation $(2,5.4)$ will now be differenced

$$
\begin{aligned}
& {\left[\Delta E_{e}^{J}\right]_{k-1 / 2, l-1 / 2}=\frac{c^{2}(\Delta t)}{8 \pi}\left[j \frac{n\left(k_{k}^{2}+z_{k}^{2}\right) \mid}{R j}\left(\frac{j i}{\partial l}\right)^{2}\right.} \\
& \quad+\left\{\frac{\pi\left(k_{l}^{2}+z_{l}^{2}\right)}{R j}-\left(\frac{\partial u}{\partial k}\right)^{2}\right]_{k-1 / 2, i-1 / 2}
\end{aligned}
$$

For conservation of energy, the joule heating should be consistent With the magnetic diffusion model, therefore the terms in the brackets $\left\{\begin{array}{l}\text { fere cast in the form } \\ \text { f }\end{array}\right.$

$$
\left\{\frac{2 \pi\left(R_{k}^{2}+z_{k}^{2}\right)}{\frac{v}{n}}\right\}
$$

But this bracket was calculated on the zone boundary for the diffusian, therefore one must calculate the energy deposited at the boundary centers and then shar: the energy with the zones common to the boundary. The sharing was based on a volume weighting of the resistivity. In a tuanner analogous to the magnetic diffusion, 
Eq. $(2.5,5)$ becomes

$$
\begin{aligned}
& {\left[\Delta \mathrm{E}_{\mathrm{e}}^{\mathrm{J}}\right]_{k-1 / 2, k-1 / 2}=B\left\{\delta_{3} W_{3}\left(\psi_{k-1 / 2, \ell+1 / 2}-\psi_{k-1 / 2, \ell-1 / 2}\right)^{2}\right.} \\
& +t_{4} l i_{4}\left(u_{k-1 / 2, \ell-1 / 2}-\psi_{k-1 / 2, \ell-3 / 2}\right)^{2} \\
& +\xi_{1} w_{1}\left(\psi_{k+1 / 2, l-1 / 2}-\psi_{k}-1 / 2, l-1 / 2\right)^{2} \\
& \left.+\xi_{2} w_{2}\left(\psi_{k-1 / 2, l-1 / 2}-\psi_{k-3 / 2, \ell-1 / 2}\right)^{2}\right\}
\end{aligned}
$$

where $\xi_{1}, \xi_{2,} \xi_{3}$ and $\xi_{4}$ were defined by Eqs, $(2,4,2 a)$ and $(2.4 .2 b)$; and

$$
\begin{aligned}
& w_{1}=\frac{(n V)_{k-1 / 2, \ell-1 / 2}}{(n V)_{k-1 / 2, k-1 / 2}+(n V)_{k+1 / 2, \ell-1 / 2}} \\
& w_{2}=\frac{(n V)_{k-1 / 2, \ell-1 / 2}}{(n V)_{k-1 / 2, \ell-1 / 2}+(n V)_{k-3 / 2, \ell-1 / 2}} \\
& w_{3}=\frac{(n V)_{k-1 / 2, \ell-1 / 2}}{(n V)_{k-1 / 2, \ell+1 / 2}+(n V)_{k-1 / 2, \ell-1 / 2}} \\
& w_{4}=\frac{(n V)_{k-1 / 2, \ell-1 / 2}}{(n V)_{k-1 / 2, \ell-1 / 2}+(n V)_{k-1 / 2, \ell-3 / 2}} \\
& B=\frac{c^{2} \Delta t}{8 \pi}
\end{aligned}
$$

The reststivity is alrady zone centered, and $V$ is calculated usirg Eq. $(2.3 .6)$.

Now the timing of the term $\Delta \mathrm{E}_{\mathrm{e}}^{\mathrm{J}}$ should be considered. It is computationally easier to calculate the magnetic diffusion $\left(\psi^{n+1}\right)$ after the energy equation is solved. Also $R, 2$, and $V$ are available at time $n+1$, 
but s-ice $n=n\left(T_{e}\right)$, it is not fully advanced in time. This is because $T_{e}$ cannot be advanced to time $n+1$ until the thermal conduction and foule heating have been considered. Overall it seems advantageous to calculate $\Delta E_{e}^{J}$ after the energy equation, and after the magnetic diffusion using $J$ at $t^{n+1}$ and $n$ at almoet $t^{n+1}$.

The joule heating is calculated as nutlined above and is added to the electron Internal energy. 


\subsection{Thermal Conduction}

The changes to the electron and fon internal energy due to thermal conduction, from Eqs. $(2,0,3)$ and $(2,0,4)$ are

$$
\frac{d E_{e}}{d t}=-\nabla \cdot \vec{Q}_{e} \text { and } \frac{d E_{1}}{d t}=-\nabla \cdot \vec{Q}_{1}
$$

where $E_{e}$ and $E_{1}$ are energy densicles, and $\vec{Q}_{1}$ and $\vec{Q}_{e}$ are the heat flux vectors. Since the electron and ion conduction equations are identical in form, the subscript w111 be dropped and a general equation of thst form w111 be discussed. The heat flux 1s defined

$$
\bar{Q}=-\kappa_{\perp} v \mathrm{~T}
$$

where only $\kappa_{\perp}$ is considered (since $\vec{B}=B_{\theta}(R, Z) \hat{\theta}$ ) and $T$ is the temperature. Using $E=\frac{3}{2} \mathrm{nKT}$, kq. (2.6.1), and dropping the $\perp$ subscript

$$
\frac{\partial T}{\partial t}=G V \cdot(\kappa V T)
$$

whera $G=\frac{2}{3 \mathrm{nK}}$, and Eq. (2.6.3) is the standard diffusion equation.

The Lagranglan difference scheme used to solve this type of equation was discussed in detall in the section on magnetic diffusion. Therefore, the differencing of Eq. (2.6.3) wlll be covered with a minimum of detalls.

Transforming to Lagranglan space, and dropping the cross terms gives

$$
\frac{\partial T}{\partial t}=\frac{G}{R j}\left[\frac{\partial}{\partial k}\left\{\frac{\left(R_{l}^{2}+z_{l}^{2}\right) R k}{j} \frac{\partial T}{\partial k}\right\}+\frac{\partial}{\partial l}\left\{\frac{\left(R_{k}^{2}+z_{k}^{2}\right) R k}{j} \frac{\partial T}{\partial l}\right\}\right](2.6 .4)
$$


Differencing as before

$$
\begin{aligned}
\frac{\partial T}{\partial t}= & Y_{1}\left(T_{k+1 / 2, \ell-1 / 2}-T_{k-1 / 2, \ell-1 / 2}\right) \\
& -Y_{2}\left(T_{k-1 / 2, \ell-1 / 2}-T_{k-3 / 2, \ell-1 / 2}\right) \\
& +Y_{3}\left(T_{k-1 / 2, \ell+1 / 2}-T_{k-1 / 2, \ell-1 / 2}\right) \\
& -Y_{4}\left(T_{k-1 / 2, \ell-1 / 2}-T_{k-1 / 2, \ell-3 / 2}\right)
\end{aligned}
$$

where $Y_{1}-Y_{4}$ are defined as

$$
\begin{aligned}
& Y_{1}=\lambda\left[\frac{\left(R_{l}^{2}+z_{l}^{2}\right) R_{k}}{j}\right]_{k, \ell-1 / 2} \\
& Y_{2}=\lambda\left[\frac{\left(R_{l}^{2}+z_{l}^{2}\right) R_{k}}{j}\right]_{k-1, \ell-1 / 2} \\
& Y_{3}=\lambda\left[\frac{\left(R_{k}^{2}+z_{k}^{2}\right) R_{k}}{j}\right]_{k-1 / 2, \ell} \\
& Y_{4}=\lambda\left[\frac{\left(R_{k}^{2}+R_{k}^{2}\right) R_{k}}{j}\right]_{k-1 / 2, \ell-1} \\
& \lambda=\frac{G}{R_{j}}
\end{aligned}
$$

and

$$
\begin{aligned}
& {\left[\frac{\left(R_{l}^{2}+z_{l}^{2}\right) R_{k}}{j}\right]_{k, \ell-1 / 2}=\frac{1}{8}\left[\left\{\frac{\left(R_{l}^{2}+z_{l}^{2}\right)}{j}\right\}_{k+1 / 2, \ell-1 / 2}\right.} \\
& \left.+\left\{\frac{\left(R_{l}^{2}+z_{l}^{2}\right)}{j}\right\}_{k-1 / 2, \ell-1 / 2}\right] \\
& \cdot\left(R_{k, l}+R_{k, \ell-1}\right)\left(k_{k+1 / 2, \ell-1 / 2}+k_{k-1 / 2, \ell-1 / 2}\right)
\end{aligned}
$$


with similar expressions for the factors in $Y_{2}, Y_{3}$ and $Y_{4}$. Using splitting, und differencing tmplicitly in time, Eq. (2.6.5) becomes

$$
\begin{aligned}
& -Y_{3} T_{k-1 / 2, \ell+1 / 2}^{n^{\prime}}+\left(1+Y_{3}+Y_{4}\right) T_{k-1 / 2, \ell-1 / 2}^{n^{\prime}} \\
& -Y_{4} T_{k-1 / 2, \ell-3 / 2}^{n^{\prime}}=T_{k-1 / 2, \ell-1 / 2}^{n} \\
& -Y_{1} T_{k+1 / 2, \ell-1 / 2}^{n+1}+\left(1+Y_{1}+Y_{2}\right) T_{k-1 / 2, \ell-1 / 2}^{n+1} \\
& -Y_{2} T_{k-3 / 2, \ell-1 / 2}^{n+1}={ }^{n}{ }_{k-1 / 2, \ell-1 / 2}^{\prime}
\end{aligned}
$$

once again, the above equat $j u n s$ are in a form easily solved by the tridiagonal method, which was discussed earlier. Solving Eqg. $(2,6.6)$ and $(2,6,7)$ gives the change in the zone temperature due to conduction.

This equation is solved twice, cuce for electrons, and once for ions, where the tramsport coefficients $k_{1 e}$, and $k_{11}$ are modeled after Braginskii ${ }^{4}$ and are discussed in Appendix 2. 
3. QUALITATIVE DESCRIPTION OF MONTE CARLO MODEL

The basic Idea of any Monte Carlo model is that the cumulative effect of many independent events can be calculated by summing over a finite number of events chosen at random but in accord with the approprlace probability distribution. Specifically, the effect of high energy charged particles on a Maxwellian fluid plasma can be calculated 1f the probabilities for all the Individual events are known. For Monte Carlo transport, one must calculate the equation of motion for a finite but representative sample of the charged particles. Since the equation of motion is very complicated, it is not solved in an analytic fashion, but is calculated by moving the Monte Carlo test bundle through the medium over many smali time steps, according to the forces acting on the bundle. During the travel of a bundle, the probability of collision is calculated, and using a random number, weighted against the probability of collision, one can force the bundle to collide with the fluid at various points along its orbit. These collisions will alter the bundle's energy and momentum, and could also result in a thermonuclear reaction.

The equation of motion for a high energy particle in a Maxwellian fluid plasma might be written

$$
\begin{aligned}
\frac{d \vec{v}}{d t}= & \frac{g}{m c} \vec{v} \times \vec{B}+\{\text { sma } 11 \text { angle collision drag term }\} \\
& +\{\text { large angle scattering term }\}
\end{aligned}
$$

where the force term $q$ t has been assumed to be small compared to the Lorentz force. This equation was solved in three steps. For a selected 
time step $(\Delta t), a \Delta \vec{v}$ and a $\Delta \vec{X}$ were calculated fron the Lorentz force. The Monte Carlo bundle was then advanced in configurational and velocity space accordingly. The change in energy due to small angle collisions for this $\Delta t$ was now calculated by integrating the equation for the energy loss rate of the energetic ions to the electron and Ion Maxwellians ${ }^{12-15}$.

$$
\begin{aligned}
& \left.\frac{d E_{1}}{d t}\right)_{e l c c}=B_{e} E_{1} \\
& \left.\frac{d E_{1}}{d t}\right)_{\text {ions }}=B_{1} E_{1}^{-1 / 2}
\end{aligned}
$$

where $E_{1}$ is the energetic ion's energy, and

$$
\begin{aligned}
& B_{i}=2 \sqrt{2 m_{1}} \pi e^{4} z_{1}^{2} \ln \Lambda_{1} \sum_{1=1}^{N} \frac{n_{i} z_{1}^{2}}{m_{1}} \\
& B_{e}=\frac{8 \sqrt{2 \pi m_{e}} z_{1}^{2} e^{4} n_{e} \ln \Lambda_{e}}{3 m_{1}\left(k{ }_{e}\right)^{3 / 2}}
\end{aligned}
$$

The derivation of these equations is discussed in Appendix 4, and their integration is developed in a following section. Thus for a given $\Delta t$, Eqs. (3.0.2) and (3.0.3) are integrated, giving a $\Delta E_{1}$, the change in the ions energy due to snall angle collistons. This $\Delta E_{1}$ yields a new $\Delta v$ (speed) and 1t is assumed that the divection does not change, 1.e., the Ion's relaxation time is less than the deflection time.

It should be noted that this solution of the small angle drag term is done deterministically and not with Nonte Carlo techniques. To calculate this term in a pure Nonte Carlo lashion would be prohibitive considering the very large number of electron-ion collisions, and probably would not be any more accurate. 
The last term, the large angle collision term is calculated in a Monte Carlo manner. Having moved the particle a $\Delta \overrightarrow{\mathrm{X}}$ in a $\Delta t$ one can calculate the probability that the particle suffered a large angle nuclear colliston or an $1 \mathrm{n}-\mathrm{f} 1 \mathrm{ight}$ thermonuclear reaction. This probability is compared to a random number, and if a collision occurs the approximation is made that it occurred at the end of the increment $\Delta \vec{X}$ at time $t+\Delta t$. It is possible to randomly allow the colliston anywhere along its incremental path, but then the small angle term and the Lorentz term would have to be raversed in time to the collision point, which is computationally very tedious.

Admittedly, the large angle collision term is small compared to the small angle term and could be dropped without significant effect, but the collisions which result in in-flight reactions may represent a very siguificant part of the energy generated for some types of pioblems ${ }^{15}$. The reason for doing the large angle scattering with the in-fight reactions was more a matter of conventence than a physics necessity. The cross section data used ${ }^{16-22}$, contained both pleces of physics and it was simple to do both.

The orbit of the high energy particle is thus calculated over many time steps until one of the following happens: 1t thermalizes (reaches $3 / 2 \mathrm{KT}_{1}$ and becomes part of the Maxwellian), It escapes beyond the boundary of the problem, or the problem reaches its concluston. Of course the ion may have an $1 n-f i l g h t$ reaction resulting in two different particles. For example: ${ }^{3} \mathrm{H}\left({ }^{2} \mathrm{H}, \mathrm{n}\right) \alpha--$ an energetic triton may collide with a Maxwe1lian deuteron resulting In a neutron and alpha particle. The neutron is assumed 
to escape with all its energy, and the alpha is scattered isotropically and followed in 1ieu of the incoming triton. The tracking of these ions is further complicated by the Lagrangian definition of the zones. The variables that affect the orbit change from zone to zone, so that the $\Delta \vec{X}$ an lon bundle moves can never be greater than the distance to a zone boundary crossing. When a bundle reaches a new zone, the appropriate cross sections, and variables are updated and the bundle tracking is resumed. The method used to track bundles in a Lagrangian mesh is covered in Appendix 5.

At the end of each $\Delta t$ in a particle's orbit, the change in the momentum and energy to the Maxwellian fluids are calculated and savec as source terms for the MHD conservation equations. When a bundle is birn from the fluid, or thermalizes, the tiass of the zone is adfusted.

This section has been a qualitative description of the Monte Carlo transport model that was used, and the following sections and the appendices will cover more of the specifics. In doing a Monte Carlo calculation the techniques used by one person on one type of computer may not be useful for another problem on a different computer. Therefore the nonphysics Monte Carlo techniques that were used (sample size determination, etc.) w1ll be discussed only brlefly in Appendix 6 . 


\subsection{Integration of the Lorentz Force}

As stated in an earlier sectlon, the first atep in solving the equation of motion, Eq. (3.0.1), was to integrate the Lorentz force, 1.e., solve the equation

$$
\frac{d \vec{v}}{d t}=\frac{g}{m c} \vec{v} \times \vec{B}
$$

This equation has been solved for particle codes by a large number of people 1,23 , and in most of these cases the plasma is considered coll1stonless. Kruer's paper ${ }^{23}$ reviews particle codes and their use in plasma simulation and gives a fafrly extensive list of further references on the subject. Since this model consider collisions and the high energy charged particles have a falriy good chance of chermalizing, one does not need the high degree of accuracy that one would want in a collisionless plasma simulation.

For a given time step (more will be said about this time step control in another paragraph in this section), Eq. (3.1.1) is differenced approximarely as follows

$$
\vec{v}_{1}^{n+1}=\vec{v}_{i}^{n}+\frac{\Delta t}{c}\left(\frac{q}{m}\right)_{1}\left[\vec{v}_{1} \times \vec{B}\right]^{n+1 / 2}
$$

where 1 denotes the ith Nonte Carlo Ion sample. The word approximately was used because the Lorentz force was not readily avallable at time $\mathrm{n}+1 / 2$, therefore a 2 pass iterative scheme was used to calculate 1t (a predictor-corrector if you prefer). Equation (3.1.2) was solved in carteslan $(x, y, z)$ space for a full 3 component magnetic fleld: $\vec{B}=B_{\theta} \hat{\theta}+B_{r} \hat{r}+B_{z} \hat{k}$. This is inconsistent with the MAD section which only had $a B_{\theta}$ component, but was programmed this way in anticipation of 
future changes to the code. For this project, $B_{r}$ and $B_{2}$ were set equal to zero. To do the tracking in cartesian space the magnetic field (in cylindrical coordinates) was decomposed to 1 ts cartesian components. The details of the cartesian tracking in a 2-D Lagranglan mesh are covered in Appendix 5. The particle tracking could probably be done in cylindrical coordinates, but it appeared to be simpler in cartesian, particularly when calculating the distance to zonal boundary crossing.

Therefore given a $\Delta t, \vec{v} \times \vec{B}$ was predicted at time $n+1 / 2$ and using this time centered Lorentz force, the velocity at time $n+1$ is calculated. The velocity is then simply integrated to get a new position coordinate

$$
\vec{x}^{n+1}=\overrightarrow{\mathrm{x}}^{n}+\Delta t \vec{v}^{n}
$$

It seemed logical that the time step for the Lorentz force integration should be controlled by the magnetic field strength. For a strong $\vec{B}$ field and tight orbits, a small $\Delta t$ would be needed and for very weak fields a large $\Delta t$ could be tolerated. Therefore the $\Delta t$ used in solving Eq. (3.1.1) was arbitrarily set such that

$$
f_{c} F \Delta t_{\operatorname{mag}} \frac{\left|\vec{v}_{1}\right|}{|\vec{v}|} \leq 1
$$

where $f_{c}=\frac{q B}{2 \pi m c}=$ the cyclotron frequency, and $F$ was an arbitrary multiplier, usually set to 20 . In other words, with $F=20$, one is allowing the ion to only move along $1 / 20$ of its local Larmor orbit. For a untform $\vec{B}$ field and a circular orbit, the ion's track would be a 20 sided polygon (icosogon). Experimentation with $F$ seemed to Indicate a value of 
20 was adequate provided a time centered second order accurate scheme (Eq. (3.1.2)) was used.

It should be noted that in the actual programming of Eq. $(3,1.2)$ other considerations may require the $\Delta t$ to be even smaller. For example one does not want the particle's orbit to carry it into another zone, but only to 1 ts boundary, therefore the time to boundary must be calculated $\left(\Delta t_{B N D R Y}\right)$.

$$
\Delta t_{B N D R Y}=\left(\vec{x}_{B N D R Y}-\vec{x}^{n}\right) / \vec{v}^{n}
$$

And in addition one must calculate the time to thermalization $\left(\Delta t_{T H}\right)-$ the time it takes for the energetic 1on to reach $3 / 2 \mathrm{KT}_{i}$, when it no longer is a Monte Carlo bundle, but becomes part of the Maxwellian Ion fluid. (See Section 3.2) for the $\Delta t_{T H}$ calculation). It is the minimum of \{ $\Delta t_{\text {MAG }}, \Delta t_{B N D R Y}, \Delta t_{T H}$ \} that is used for the tracking of the ion.

Now that the Nonte Carlo Ion bundle has been advanced In velocity and configurational space due to the Lorentz force, the energy and momentum losses to the bundle (gains to the fluid plasma) are calculated for that time step. 


\subsection{Integration of Small Angle Collision Term}

The object of this section is to develop the equations used to calculate the energy loss to an energetic ion as it cravels through a Maxwellian fluld of electrons and 1ons. Only small angle collisions are considered in this section and the approximation is made that the equilibration time of the energet ic ion is less than the $90^{\circ}$ deflection time. Therefore a stralght line path is assumed. The energy $10 s 8$ of the energetic lons is given to the Maxwellian fluids. The fractional amounts given to each are also calculated,

From Append $1 x$ the energy loss rate equations were derived as (Note: zinus sign dropped)

$$
\begin{aligned}
& \left(\frac{d E_{1}}{d t}\right)_{e}=\frac{32 n_{e} z_{1}^{2} e^{4}}{3 m_{1}<v_{e}^{>}}\left(\frac{E_{1}}{k r_{e}}\right) \text { in } \Lambda_{e} \\
& \left(\frac{d E_{1}}{d t}\right)_{1}=\frac{4 \pi e^{4} z_{1}^{2} \text { In } \Lambda_{1}}{v_{1}} \sum_{1=1}^{N} \frac{n_{1} z_{1}^{2}}{m_{1}}
\end{aligned}
$$

where the 1 subscript refers to the energetic ion, the e subscript to the electron fluid, and the 1 subscript to the lon fluld. Letting

$$
\left.<v_{e}\right\rangle=\sqrt{\frac{8 k T_{e}}{\pi m_{e}}} ; v_{1}=\sqrt{\frac{2 E_{1}}{m_{1}}}
$$

E.qs. (3.2.1), (3.2.2) become

$$
\left(\frac{d E_{1}}{d t}\right)_{e}=B_{e} E_{1}
$$




$$
\begin{aligned}
& \left(\frac{d E_{1}}{d t}\right)_{1}=B_{1} E_{1}^{-1 / 2} \\
& B_{e}=\frac{8 \sqrt{2 \pi m_{e}} z_{1}^{2} e^{4} n_{e} \ln \Lambda_{e}}{3 m_{1}\left(K T_{e}\right)^{3 / 2}} \\
& B_{1}=2 \sqrt{2 m_{1}} \pi e^{4} z_{1}^{2} \ln \Lambda_{1} \cdot \sum_{i=1}^{N} \frac{n_{1} z_{1}^{2}}{m_{1}}
\end{aligned}
$$

Equation $(3.2 .3)$ is the energy loss rate of an energetic ion in a Maxwellian of electrons where $v_{1} \ll\left\langle v_{e}\right\rangle$. Equation (3.2.4) 1s the energy loss rate of an energetic ion in a Maxwellian of lons where $v_{1} \gg v_{1}$.

The total energy loss rate is just the sum of (3.2.3) and (3.2.4)

$$
\frac{d E_{1}}{d t}=B_{e} E_{1}+B_{i} E_{1}^{-1 / 2}
$$

With a little algebra, Eq. (3.2.5) becomes

$$
d t=\frac{E_{1}^{1 / 2} d E_{1}}{\beta_{1}\left(1+B_{T} E_{1}^{3 / 2}\right)}
$$

where $B_{T}=B_{e} / B_{I}$. Integrating

$$
\Delta t=\int_{E_{f}}^{E_{s}} \frac{E_{1}^{1 / 2} d E_{1}}{\beta_{i}\left(1+B_{T} E_{1}^{3 / 2}\right)}
$$

where $E_{S} 1 s$ the energy of the energetic Ion at the start of the time step and $E_{f}$ is the energy at the end of $\Delta t$. Since $B_{i}$ and $B_{e}$ are not functions 
of $E_{1}, E_{q},(3.2 .7)$ is easily integrated

$$
\Delta t=\frac{2}{3 \beta_{e}} \ln \frac{\left(I+\beta_{T} E_{s}^{3 / 2}\right)}{\left(1+\beta_{T} E_{f}^{3 / 2}\right)}
$$

This is a very useful equation. We know $E_{s^{\prime}} B_{T}$, and $B_{e}$, and if $E_{f}$ is known we can calculate the $\Delta t$ needed to reach $E_{f}$. or if $\Delta t$ is known, then $E_{f}$ can be calculated. Equation $(3.2 .8)$ is easily rearranged to give the equation for $E_{f}$

$$
E_{f}=\left\{\frac{1}{\beta_{T}}\left[\left(1+\beta_{T} E_{S}^{3 / 2}\right) e^{-\frac{3 \beta_{e} \Delta t}{2}}-1\right]\right\}^{2 / 3}
$$

As was stated earlier, the $\Delta t$ to be used in the Monte Carlo tracking must be less than the time it takes for the fon to reach thermalization. Therefore Eq. $(3.2 .8)$ is used to calculate this $\Delta t_{T H}$

$$
\Delta t_{T H}=\frac{2}{3 \beta_{e}} \ln \frac{\left(1+\beta_{T} E_{s}^{3 / 2}\right)}{\left(1+\beta_{T} E_{T H}^{3 / 2}\right)}
$$

where $E_{T H}=3 / 2 K T_{i}$; a known quant1ty. Having calculated $\Delta t_{M A G}$ (Eq. 3.1.4). and $\Delta t_{\text {BNDRY }}$ (Eq. 3.1.5), and $\Delta t_{T H}$ (Eq. 3.2.10), the minimum of these three is selected and used to advance the Monte Carlo bundle. This $\Delta t$ is substituted into Eqs. (3.1.2) and (3.1.3) to advance the bundle and into Eq. (3.2.9) to calculate its final energy. The total energy change is simply

$$
\Delta E_{1}=E_{s}-E_{f}
$$


Now we must calculate that fraction of $A E_{1}$ which goes to the fluld Ions and that which goes to the electrons. The energy given to the Maxwellian flutd lons ig given by

$$
\left.\Delta E_{1}\right)_{1}=\int_{E_{f}}^{E_{g}}\left(\frac{d E_{1}}{d t}\right)_{1} d t
$$

Using Eqs. $(3.2 .4)$ and $(3.2 .6)$, the above becomer

$$
\left.\Delta E_{1}\right)_{1}=\int_{E_{f}}^{E_{s}} \frac{d E_{1}}{\left(1+B_{T} E_{1}^{3 / 2}\right)}
$$

This can $b \geq 1$ integrated and gives

$$
\left(\Delta E_{1}\right)_{1}=\left.\frac{2}{3 B_{1} g}\left[\frac{1}{2} \ln \frac{g^{2}-g x+x^{2}}{(g+x)^{2}}+\sqrt{3} \tan ^{-1}\left(\frac{2 x-g}{g \sqrt{3}}\right)\right]\right|_{E_{t}} ^{E_{s}}
$$

where $g=\left(1 / \beta_{T}\right)^{1 / 3}$ and $x=E_{1}^{1 / 2}$. Th1s is computationally tedious and $1 t$ Is simpler to use the approximation 17

$$
\begin{aligned}
& \left(\Delta E_{1}\right)_{1}=\left(\frac{1}{\beta_{T}}\right)^{2 / 3} F(u) \\
& F(u)=\int_{u_{f}}^{u_{s}} \frac{d u}{u^{3 / 2}+1}
\end{aligned}
$$

where $u^{3 / 2}=B_{T} E^{3 / 2}$ and $F(u)$ can be approximated by

$$
F(u)= \begin{cases}u-.25 u^{2} & , u \leq 1 \\ 2.4-2 u^{-1 / 2}+.35 u^{-1}, & u>1\end{cases}
$$


Th1s set of equations is used to calculate the fraction of energy that goes to the fons. From conservation of energy the remaining energy goes to the electrons

$$
\left(\Delta E_{1}\right)_{e}=\Delta E_{1}-\left(\Delta E_{1}\right)_{1}
$$

Now the speed of the energetic ion is decreased to be consistent with its new energy. Note that the direction is not altered.

$$
\begin{aligned}
& v_{x}^{n^{\prime \prime}}=v_{x}^{n^{\prime}} \frac{|\vec{v}|^{n^{\prime \prime}}}{|\vec{v}|^{n^{\prime}}} \\
& |\vec{v}|^{n \prime}=\sqrt{\frac{2 E_{f}}{m_{1}}}
\end{aligned}
$$

etc. for $y$ and 2 components; where $n^{\text {"t }}$ refers to the time after the strali angle collisions (but before any large angle collision) and $n^{\prime}$ refers to time at the end of the Lorentz motion (but before large and small angle collisions).

The completes the integration of the sthall angle collision term, but a summary might clarify the procedure. The following is a word description of the flow of the Monte Carlo calculation up to this point.

1. A $\Delta t_{\text {MAS }}$ is calculated from Eq. (3.1.4).

2. A $\Delta t_{T H}$ is calculated from Eq. $(3.2,10)$.

3. $\triangle \Delta t_{B N D R Y}$ is calculated from Eq. (3.1.5),

4. Select minimum of $\left\{\Delta t_{M A G}, \Delta t_{T H}, \Delta t_{B N D R Y}\right\}$.

5. Calculate $\Delta \vec{v}$ from Eq. (3.1.2).

6. Calculate $\Delta x$ from Eq. (3.1.3).

7. Calculate $\Delta E_{1}$ from Eq. (3.2.11).

8. Calculate $\left(\Delta E_{1}\right)_{i}$ from Eq. (3.2.12). 


\section{Calculate $\left(\Delta \mathrm{E}_{1}\right)_{\mathrm{e}}$ from Eq. (3.2.13). \\ 10. Calculate $|\vec{v}|$ from Eq. (3.2.14).}

The large angle collisions can now be calculated. 


\subsection{Integration of the Large Angle Collision Term}

In the last two sections the Monte Carlo bundle was advanced in velocity and configuration space due to the Lorentz force, and the speed and energy were updated from smali angle collomb collisions. Now the probability of the Nonte Carlo bundle to have a large angle nuclear scattering, or an in-flight nuclear reaction over the speciffed time step (At) can be calculated. The crosg sectlons and data used were adapted from papers written by Corman and others. 16-22 only the following scatterings and reactlons were considered:

1. Deutfions: an energetic deuceron was able to undergo the followling in-flight reactions

$$
\begin{aligned}
& d+d+n+{ }^{3} \mathrm{He} \\
& d+d \rightarrow p+t \\
& d+t \rightarrow n+a
\end{aligned}
$$

and could scatter elastically from other Maxwellian deuterlum 1ons. Elastic scattering of deuterons on tritium was not modeled.

2. Tritons: energetic triton's were allowed to have an in-flight reaction with Maxwellian deuterium; Eq. (3.3.3).

3. Alphas: energetic alpha particles could undergo nuclear elastic scatterings with Maxwellian deuterium 1ons. Scactering of alphas on tritium was not calculated. 
The only reason for not considering elastic collisions on tritium was that the cross section daca was not readily available, and the relative unimportance of large angle scattering to the overall problem didn't justify spending the time needed to complle $1 t$.

In all cages above, the probability for an event happening over the given $\Delta t$ is given by the following general type of equation.

$$
P_{1}=1-\exp \left[-\Delta t \sum_{j=1}^{N} n_{j}(\sigma v)_{i-j}\right]
$$

where $i$ denotes the energetic particle and $f$ the Haxwellian spectes It is colliding and reacting with. The term ( $\sigma \mathrm{V})$ is replaced by <ov>, the Maxwell averaged cross section, for the in-flight reactions. The cross sections are tabulated in the code as a function of the test ion energy and plasma fon temperature. As an example of Eq. (3.3.4), the probabllity of an energetic deuteron event (case I above) is

$$
\begin{aligned}
P_{d}= & 1-\exp \left[-\Delta t\left(n_{d}\langle\sigma V\rangle_{d-d}^{(1)}+n_{d}\langle\sigma V\rangle_{d-d}^{(2)}\right.\right. \\
& \left.\left.+n_{t}\langle\sigma V\rangle_{d-t}+n_{d}(\sigma V)_{d-d}^{(s)}\right)\right]
\end{aligned}
$$

where the superscripts (1) and (2) refer to the different branches of the d-d reaction, and the (s) refers to the elastic scattering cross section. The data and equations needed to determine $\langle\sigma \mathrm{V}\rangle$ and (oV) are given in the references mentioned earlier.

Having calculated the total probability for an event using Eq. (3.3.4), one now compares this with a random number. If the random number is less 
than $P_{i}$, then an event is assumed and a second random number is selected and compared to the individual probabilities to decide which event occurred. For simplicity it is assumed the event takes place at the end of the $\Delta t$. The event could have taken place anywhere along its elemental pach, but this would require "backing up" the Lorentz force and the small angle collision terms to the colliaion point; which would be very tedious.

When the event is an in-flight reaction, then the reaction products are created and scattered 1sotropically with their appropriate energies. When the event is an elastic scatter, the scattering angle must be randomly determined from a table. This table of angles was generated in the usual fashion, by integrating the differential cross section (which Is a function of the test lons energy) over a given set of angles and equating the results to a set of random numbers. Thus given a random number and the energy of the test ion, a scattering angle is easily determined. Using conservation of energy and momentum, the energy and remaining angles of the scatter for the test and target particles are easily calculated using the usual center of mass equations for elastic scattering.

It is interenting to note that after an event, one may be left with two high energy bundles to track. All high energy neutrons generated from the reactions are assumed to escape immediately with their energy. But any resultant charged particles from a reaction or, the scattered test particle and its scattered target particle must be tracked as Monte Carlo particles until they reach thermalization energy. Thus particles 
may $b \in$ scattered out of the Maxwellian model into the Monte Carlo model, and vice versa.

This completes the Monte Carlo time step. The particle tracking continues for another $\Delta t$ until the sum of the Monce Carlo At's equals an MHD St, at which time the Nonte Carlo bundles are stored on diac (census) until after the MHD has been advanced a time step. 


\subsection{Momentum Conservation}

The last three sections have described how the equation of motion for a Monte Carlo bundle of energetic ions was solved. It Involved three computational steps: 1 , the integraction of the Lorentz force, 2 , Integration of the small angle coulomb collision term, and 3 . Integration of the large angle scattering and in-flight reaction term. In addition to calculating the energetics of these processes, one must also calculate the momentum changes between the fluid and the Monte Carlo system of particles. Changes in the fluid's momentum from the Monte Carlo processes are saved as a source term and added to the MHD momentum equation (Eq. $(2,0,2)$ ). This source cerm is of the form

$$
(\overrightarrow{\vec{V}})_{\mathrm{MC}}=\left(\frac{\Delta \overrightarrow{\mathrm{V}}}{\Delta \mathrm{t}}\right)_{\mathrm{MC}}=\left(\frac{{\overrightarrow{\hat{n}^{n+1}}}^{\mathrm{n}}-\overrightarrow{\mathrm{V}}^{\mathrm{n}}}{\Delta \mathrm{t}}\right)_{\mathrm{MC}}
$$

where $\vec{\nabla}$ is the fluid velocity. Thus one needs to know what the fluids final velocity would be as a result of the Monte Carlo processes. The tern $(\overrightarrow{\hat{v}})_{M C}$ is the resultant of the sur of all processes for all Monte Carlo bundles. Note that the fluld velocity is not changed after each Monte Carlo event, but is changed only through the Monte Carlo momentum term in the MHD monentum equation.

\section{A. Lorentz Force}

When integrating the Lorentz force, the particle's velocity vector was rotated, but the speed and energy remained constant. Since the vector veloctty has changed, the momentum of the bundle has changed at the expense of the momentum of the magnetic field. One might assume that the fluld and field are strongly coupled, therefore the momentum change ought 
to be given to the fluid. But this creates a problem. If the fluid is to receive the momentum, then by conservation, one can't hold the particle energy constant over the Lorentz rotation unless the energy is to come from the magnetic field. The physics of this three component process was not understood, so the assumption was made that

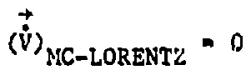

1.e., the Lorentz rotation of the Monte Carlo bundle contributed no momentum changes to the fluid.

\section{B. Small Angle Coulomb Collisions}

The calculation of momentum exchange from small angle coulomb collistons was modeled by considering the collision between a zone of mass $N$ and velocity $\vec{V}$ with a particle of mass $m$ and velocity $\vec{v}$. The collision kinematics are conveniently described in the center of mass (momentum) coordinates. Letting the subscript CM denote a variable in the center of mass coordinates, the following can easily be derived

$$
\begin{aligned}
& \vec{v}_{C M}=\left(\frac{M}{m+M}\right)(\vec{v}-\vec{v}) \\
& \vec{v}_{C M}=\left(\frac{m}{m+M}\right)(\vec{v}-\vec{v})
\end{aligned}
$$

and $\vec{U}_{\ell}$, the CM velocity in the laboratory is

$$
\overrightarrow{\mathrm{U}}_{\ell}=\left(\frac{M}{m+M}\right) \vec{v}+\left(\frac{m}{m+M}\right) \vec{v}
$$

The kinetic energy of the system in the CM Is given by the equation

$$
(\mathrm{KE})_{\mathrm{CM}}=\frac{1}{2} m v_{\mathrm{CM}}^{2}+\frac{1}{2} \mathrm{~N} \mathrm{v}_{\mathrm{CM}}^{2}
$$


and using Eqg. $(3.4 .2)-(3.4 .4)$ it becomes

$$
(\mathrm{KE})_{\mathrm{CM}}=\frac{1}{2}\left(\frac{\mathrm{m} M}{m+\mathrm{M}}\right)\left|\overrightarrow{\mathrm{v}}_{\mathrm{CM}}-\overrightarrow{\mathrm{v}}_{\mathrm{CM}}\right|^{2}
$$

Letting the superscripts $n$ and $n+1$ refer to the time at the start and end of the Monte Carlo time step; assuming stralght line slowing down (scattering angle of the energetic ion is zero) and invoking conservation of energy and momentum gives

$$
\nabla^{n+1}=\mu \nabla^{n}+(1-\mu) \nabla_{\ell}^{n}
$$

where

$$
\mu=\left[\frac{(K E)_{C N}^{n+1}}{(K E)_{C N}^{n}}\right]^{1 / 2}
$$

The object is to now determine the cylindrical components of Eq. (3.4.6) by taking the dot product of $\vec{v}^{n+1}$ with the unit vectors, $\hat{r}, \hat{\theta}, \hat{z}$. Note that $v_{\theta}^{n}=0$, but $v_{\theta}^{n}$ is not, therefore a $v_{\theta}^{n+1}$ is generated. From symmetry there should be cancellation of the various $v_{\theta}^{n+1}$ components for each particle. Thus there will be no $\hat{\theta}$ component of momentum, but there will be an energy deposited to the fluid from this $v_{\theta}^{n}$ component. Thus the final lab components of the fluid velocity are

$$
\begin{aligned}
& v_{R}^{n+1}=\left[\mu+(1-\mu) \frac{M}{m+M}\right] v_{R}^{n}+(1-\mu)\left(\frac{m}{m+M}\right) v_{R}^{n} \\
& v_{z}^{n+1}=\left[\mu+(1-\mu) \frac{M}{m+M}\right] v_{z}^{n}+(1-\mu)\left(\frac{M}{m+M}\right) v_{z}^{n} \\
& v_{\theta}^{n+1}=(1-\mu)\left(\frac{m}{m+M}\right) v_{\theta}^{n}
\end{aligned}
$$


Now the Monte Carlo momentum deposiclons for small angle coulomb collistons can be calculated using Eqs. (3.4.1) and (3.4.8)

$$
\begin{aligned}
& (\vec{v})_{\text {MC-SMALL ANGLE }}=\hat{r}\left\{\left[\mu+(1-\mu) \frac{M}{m+M}\right] v_{R}^{n}\right. \\
& +(1-\mu) \frac{m}{m+M} v_{R}^{n}-v_{R}^{n}++\hat{z}\{\mu+ \\
& \left.\left.(1-\mu) \frac{M}{m+M}\right] v_{z}^{n}+(1-\mu)\left(\frac{m}{m+M}\right) v_{z}^{n}-v_{2}^{n}\right\}
\end{aligned}
$$

Note that $v_{R}, v_{z}$, and $v_{\theta}$ are to be calculated from the cartestan components $v_{x}, v_{y}$, and $v_{z}$ and their direction cosines which are used In the tracking (see Appendix 5). The components of Eq. (3.4.9) thus represent axial and radial momentuin depositions to the fluld from small angle coulomb collisions.

Since both energy and momentum must be conserved, a few words are In order concerning the energy depositions calculated in Section 3.2. The terms $\left(\Delta E_{1}\right)_{e}$ and $\left(\Delta E_{1}\right)_{1}$ calculated from Eqs, $(3.2 .12)$ and $(3.2 .13)$ must be slightly modified. The equations to calculate the energy loss rate assumed the Maxwellian fluid was at rest, but since it is not the terms $\left(\Delta E_{1}\right)_{1}$ and $\left(\Delta E_{1}\right)_{e}$ should be calculated in the fluid rest frame before using the equations of Section 3.2. This is simply done by subtracting $\vec{f}$ from $\vec{v}$ and calculating $E_{1}$ using the rest frame speed.

Thus the energy deposited to the fluid internal energy is the difference between the $(\mathrm{KE})_{\mathrm{CM}}$ over the time step, plus the energy 
deposited due to the $v_{\theta}^{n}$ component of velocity (see Eq. 3.4.8c).

$$
\Delta E=(K E)_{C M}^{n}-(K E)_{C M}^{n+1}+\frac{1}{2} M\left[(1-\mu) \frac{m}{m+M} v_{\theta}^{n}\right]^{2}
$$

The term (KE) ${ }_{\mathrm{CM}}$ is related to the kinetic energy of the Monte Carlo bundle in the fluld regt frame by the relation

$$
(\mathrm{KL})_{\mathrm{CM}}=\frac{M}{m+M}\left(E_{1}\right)_{\mathrm{fluid}} \text { rest frame }
$$

Thus knowing $E_{1}$ in the above equation, we calculate (KE) ${ }_{C M}$ for time $n$ and $\mathrm{n}+1$ and tien calculate $\mathrm{u}$ In Eq. (3.4.7). Now the amount of energy given to the internal energy of the electron and ion flulds is

$$
\begin{aligned}
& (\Delta E)_{i}=\frac{M}{m+M}\left(\Delta E_{1}\right)_{1}+f_{1} E_{\theta} \\
& (\Delta E)_{e}=\frac{M}{m+M}\left(\Delta E_{1}\right)-(\Delta E)_{1}+\left(1-f_{1}\right) E_{\theta} \\
& f_{1}=\frac{\left(\Delta E_{1}\right)_{1}}{\left(\Delta E_{1}\right)} \\
& E_{\theta}=\frac{1}{2} N \quad(1-\mu) \frac{m}{m+M} v_{\theta}^{n}
\end{aligned}
$$

where $\left(\Delta E_{1}\right)_{i}$ is from Eq. $(3.2 .12),\left(\Delta E_{1}\right)$ from Eq. $(3.2 .11)$, remembering that $E_{1}$ is in the fluid rest frame.

\section{Large Angle Scattering and In-FIlght Reactions}

When an elastic nuclear scattering event takes place the CM scattering angle is randomly selected from the approprlate distribution. Thus the collision kinematics between the Maxwellian ion (which is assumed to be at rest) and the Nonte Carlo bundle are easily done using the usual collision equations for an elastic scatter in the CM coordinates. 24 Thus the target particle is klcked out of the Maxwellian and becomes a 
Monte Carlo particle. Nomentum is conserved between the test and target particles, therefore the zone fluld does not recelve any momentum from the collision. For an in-flight reaction the reaction products are created at their appropriate energies and no momentum is given to the fluid. Therefore no momentum from large angle scattering and in-filght reactions 18 given to the flutd; $1 . e$. ,

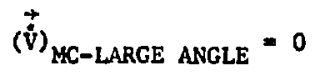

\section{Summary}

Thus in summary the only contribution to the fluid momentum is from the small angle coulomb collistons, as given by Eq, (3.4.9). And the energy terms of Section 3.2 must be modifled as in Eq. (3.4.12) - (3.4.15) to be consistent with this momentum deposition. 


\subsection{Monte Carlo Current}

As indicated in the introduction and in Figure 1, a Monte Carlo current $\left(\vec{J}_{M C}\right)$ term should be calculated and used in calculating the magnetic field. This current could be added to the current in Ampere's Law. However, in this study, $J_{M C}$ was assumed to be zero. The values of $\vec{J}_{\text {MC }}$ could be calculated by tally ing the fluxes of electrons and lons as they move through the mesh.

$$
J_{M C}=\left(J_{M C}\right)_{1}+\left(J_{M C}\right)_{e}
$$

or

$$
\vec{J}_{M C}=n_{i} z_{i} e \vec{v}_{i}-n_{e} e \vec{v}_{e}
$$

The determination of $\left(J_{M C}\right)_{1}$ is very simple. One simply counts the Monte Carlo ion bundles that traverse the zone. However calculating $\left(J_{M C}\right)_{e}$ is not so obvious as there are no Nonte Carlo clectrons. Because of the coulomb forces generated, the Ion will tend to drag electrons along with 1t. If the electrons were not dragged along, chen one would not have local charge neutrality.

In the absence of a $\vec{B}$ fleld the assumption $\vec{J}_{M C}=0$ seems quite reasonable for hydrodynamic time scales. However when there is a strong $\vec{B}$ field, the gradient and curvature drift complicate the picture as they tend to separate the ion and its companion electrons. The picture is further complicated by the fact the companion electrons will be of much lower energy than the fon and will have a much shorter mean free path, thus suffering many collistons whlle moving with the 1on. Despite thest complications, one would think that the coulomb attractive force rould 
dominate and that the Monte Carlo lons would move as neutrals; 1.e., they would have companion electrons with them. Thus $f_{N C}$ was assumed to be eero in this study.

Assuming one did know how to calculate a $J_{M C}$, then the question arigns as to what to do with 1t. One simple suggestion would be to calculate the change in $B$ due to $\mathrm{J}_{M C}$, by solving Ampere's Law, $\nabla \times \vec{B}=\frac{4 \pi}{c} \vec{J}_{M C}$, expl1c1tiy. This may (hithout care) cause instabilites, but it would be a simple way to account tur a Monte Carlo current in the MHD equations. A self-consistent scheme is described by $T$. Johnson in his Ph.D. thesis. 25 


\section{CONCLUSION}

In the preceding pages a model for simulating the transport of nonMaxwelitan Ions in a Maxwellian fluid plasmo has been developed. The non-Maxwellian ions were modeled using a Monte Carlo model and the Maxwellian fluid with a MDD model. The non-Maxwellian Ions suffered small angle coulonib collisions, large angle ocatterings, and in-flight reactions whtle orbiting in the plasma under the influence of a magnetic field. Energy and momentum depositions to the Maxwellian flulds from Monte Carlo collistons were added to the electron and Ion conservacion equations as source terms. The model was developed in a two-dimensional $(R, Z)$ coordinate system, with a Lagranglan representation for the MaD equations. A $B_{\theta}$ magnetic field with a stmple Ohn's law was assumed. A two temperature model was used, with a periect gas equation of state, a scalar pressure and a tensor artificlal viscosity. Electron and fon therma I conduction was also calculated, All physics and transport coefficients are calculated assuming a fully ionized gas, and a bremsstrahlung radiation loss term is Included to simulate the free-free transitions. The MHD conservation equations were differenced explicitly and the diffusion type equations (magnetic and thermal conduction) were differenced Implicitly.

The model was developed primarily to study the effect of thermonuclear reaction products upon a dense hut plasma. The Monte Carlo model Is particularly useful when the Larmor radil of the nun-Maswellian lons are comparible in size to the problem size. A diffusion transport model is not valid in this regime. Of course, when the magnetic field is strong and the orbits are small, the Monte Carlo model becumes too 
expensive and impractical to use. The assumption of a perfect gas and full Ionfzation are valid for hot plasmas that are burning, but must be used with caution. One would not want to simulate the magnetohydrodynamics of a cold, partially Ionized gas with this model. The Lagrangian fluid equations are useful for problems that may have multiple fluid species or a plaema that is Interacting with a wall or liner. On the other hand the hydro motion must not be so turbulent that the mesh distorts and bowties to the point where one can't run. In other words, the model should be used for hot systems that are close to hydrodynamic equilibrlum, but where the non-Maxwellian energy and momentum depositions from the thermonuclear reactions will affect the motion of the fluid.

The Monce Carlo model might also be useful for beam-injection and/or Fokker-Planck type studies.

The model was used to calculate the thermonuclear alpha particle heating of a gas-embedded 2-pinch. The problem description and results are described in decafl in Appendix 1. In summary, the results of this test calculation show that the nonlocal energy depositions of the alphas to the pinched gas result in nonunlform spattal heating and significantly lower drift losses. Desplte these differences, the overall heating and resulting expansion are in fair agreement with other computer studies. With respect to the computer model, the conclusion can be drawn that Monte Carlo is a valid calculational tool for certain plasma physics studies.

Three problems were run; all were initially identical except that each had different currents (different circuit parameters). Two of the 
problems (the smaller of the currents) ran without any difftculties, but the problen with the largest current created large B flelds and very small time steps in the Monte Carlo, Problem 3 (MC-3) nould probably be betcer run on a diffusion model. Each problem uged about 6 hourg of 7600 computer time, and each problem generated and followed approximately

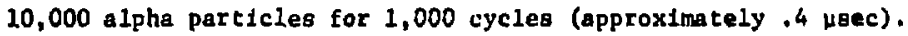

The usefulness of the model could be oignificantly Improved with the addition of the $K$ and $Z$ components of the magnetic fleld. This was essentially done in the Monte Carlo section, but were set to zero to be consiscent with the MUD. Other possible improvements would be the Inclusion of partially lonized gas phystcs, a non-perfect equation of state, and a better Ohm's law. Any of these additions could be made to che model within the current configuration of the code. Another destrable change would be to add the $\theta$ dependence to the model, but this would require redolng the Monte Carlo tracking scheme - a difficult task. The assumed $\theta$ symmetry greatly simplified the tracking by allowing one to perfortn a coordinate transformation to track in a plane. Without $\theta$ symmetry a new tracking model must be developed.

Although there $1 \mathrm{~s}$ room for improvement in this model, the concept of a Monte Carlo-MHD hybrid code has been shown to be useful. The model w111 a1d in the understanding of plasma behavior and the transport properties within a hot dense magnetized gas. 
APPENDIX 1: TEST PROBLEM - ALPHA PARTICLE HEATING OF A Z-PINCH

To test the model developed in this report, a study was made of the alpha particle heating of a z-pinch. Hartman and others have reported on their Investigation and their modeling of the alpha particle heating of a gas-embedded Z-pinch ${ }^{26,27}$ using a I-D MAD code. 28 It was decided to repeat the calculation using this MHD/Monte Carlo model.

The problem reported by Hartman was essentially as follows. A onedimensional D-T plasma (50\%-50\%) of radius 10 centimeters with $\mathrm{T}-1 \mathrm{eV}$ for $r \leq .047 \mathrm{~cm}$; and $T=$ room temperature for $.047<R \leq 10 \mathrm{~cm}$, and $p=$ $.1 \mathrm{gm} / \mathrm{cm}^{3}$ for all $\mathrm{r}$ was subdivided (zoned) in 40 zones. The Inner reglon of the problem was heated up to $T=390 \mathrm{eV}$ in $2.25 \mu \mathrm{s}$ by a resiotive pinch preheat. At $t=2.25 \mu \mathrm{s}$ a capacitor $(c=.1 \mu \mathrm{fd})$ with internal Inductance $I=50$ nhenry, charged to $V=50 \mathrm{MV}$ was switched on and crowbarred at peak current $I=30 \mathrm{MA}$. The plot of the current as a function of time Is given by curve $\| 1$ in $F 18.5 .1 .1$. The D-T reaction rates were calculated as a function of the material temperature, T. The alpha particle heating was calculated assuming that the alpha particles lose energy to the plasma by classical collisions with time constant $\tau_{c}$ and are lost out the end of the pinch by $\nabla B$ drift with time constant $\tau_{D}$ " The alpha particle lifetime for each zone was glven by $1 / \tau_{\alpha}=1 / \tau_{c}+1 / \tau_{D}$. In the loss due to drift calculation, the axial dimenston of the plasma was assumed to be $10 \mathrm{~cm}$. The alpha particle energy heated the plasma from 10 to $50 \mathrm{keV}$ and in consequence the pinch expanded radially.

Now to describe the problem as it was run on this MHD/Monte Carlo model. Although the code written to simulate this model is as yet 
unnamed, it will be referred to as the MC code. The code used by Hartman is called MAGPIE.

Since the MC model assumed a perfect gas and full lonization it was decided to not try and run the entire problem as was done on MAGPIE, but to pick up (link) the problem from MGPLE at $t=2.3 \mu s$. This was after preheat, but before the final pinch and thermonuclear burn. Only the hot plasma (inner 19 zones) was linked to MC and the remaining 21 zones were hydrodynamically simulated using a time varying pressure profile on the outer boundary of the mesh. Since MC 1s two-dimensional, It was possible to specify the axial dimension of $10 \mathrm{~cm}$. To compare with MAGPIE and to keep the MC Lagrangian zones from sausaging (and becoming bowtled), the radial accelerations were forced to be constant along a line of constant $z$. Thus the problem was essentially one dimensional in the MHD but the Nonte Carlo was still two-dimensional.

Three problems were run on MC. They all had identical initial conditions, but the current profile as a function of time was different. Problem MC-1 was run with the current given by curve \#1, F1g. 5.1.1; MC-2 with current $\$ 2$ and MC-3 with current \#3. At currents much higher than \#3, the MC code became too expensive to run since the Larmor orbits became very small and hence the Monte Carlo magnetic time step $\left(\Delta t_{\text {NAG }}-E q \cdot(3.1 .4)\right)$ became prohibltively small.

Before describing the results a few words about the phystcs differences between the MC and MAGPIE codes should be made. The key difference is the model describing the transport of the alpha particles, 
which has already been described. Another possibly significant difference is that $M C$ is a two temperature code $\left(T_{e}\right.$ and $T_{i}$ ) while MAGPIE has only a single temperature I. Both codes calculated thermal. conduction, and for times greater than 2.3 is and $r<.2 \mathrm{~cm}$ (firat 19 zones of NAGPIE) the full lonization and perfect gas assumptlons of MC are probably valid. Both codes had a radiation losg term and 1.gnored the neutron energy from the D-T reactions.

It is important to note that the problems labeled MC-1 and MAGPIE had Identical currents, therefore should be comparable. MC-2 had a smaller current and MC-3 \& larger current.

When comparing MC-1 and MAGPIE in Figs, 5.1.2-5.1.9, one sees that the results are reasonably similar in most respects (within a factor of 2). The biggest discrepancy seems to be the energy iost to the problem from drift. NAGPIE's drift energy was approximately 8 times larger than MC-1. In MC-1 the energy is not drifting out of the problem as fast as it did in MAGPIE. The lower drift can partly be attributed to a smaller gradient in the magnetic fleld (off axis) in $M C-1$ and also due to the higher densities and lower temperatures (on axis) one sees in $M C-1$. The temperature and density differences can significantly reduce the time it takes a particle to thermalize (and hence reduces the chance to escape). For example a $3.5 \mathrm{MeV}$ alpha particle in $\mathrm{D}-\mathrm{T}$ at density $.01 \mathrm{gm} / \mathrm{cm}^{3}$ and $\mathrm{T}_{\mathrm{e}}=$ $40 \mathrm{keV}$ (MAGPIE conditions) will thermalize in approximately .1 $\mu \mathrm{s}$, whereas at $I_{e}=20 \mathrm{keV}$ and $\rho=.02 \mathrm{gm} / \mathrm{cm}^{3}$ (MC-1 conditions) the time to thermalization is approximately .03 $\mu \mathrm{s}$. These $\nabla B, \rho$, and $T_{e}$ differences can easily justify the factor of 8 difference in the drift energy loss. 
The scenario in MC-1 as compared to MAGPIE seems to be the following. The pinch in MC-1 is more effective, resulting in higher initlal densities and higher radiation loss; thermonuclear burn starts similarly, but in MC-1 it tends to be off axis (about $r=.05 \mathrm{~cm}$ ), whereas in MAGPIE it is fairly uniform. This is probably caused by the lower $B$ field on axis, which doesn't trap the alpha particles as effectively as the stronger $B$ field off axis. Both problems overall have similar energy deposition from the alphas, but MC-1 Inftially loses more energy from bremsstrahlung. Later in time the MC-1 seems to expand faster causing a large drop-off in density at $r=.1$. The large difference in loss due to drift is probably explained by higher inttial densities, lower temperatures, and a significantly smaller gradient in the B field. See Figs. 5.1.7-5.1.9 for MC-1 and MAGPIE comparisons of $B, T_{e}, T_{1}, T$, and $\rho$ at $2.6 \mu s$.

It should be noted that MC-3 was not run to completion (2.7 $\mu \mathrm{s})$ because it was becoming too expensive in terms of computer time (for a scudent running a test problem). The increased current caused better pinching and better burn, but the large $B$ fleld $\left(\approx 10^{8}\right.$ gauss) created very small Monte Carlo time steps. 


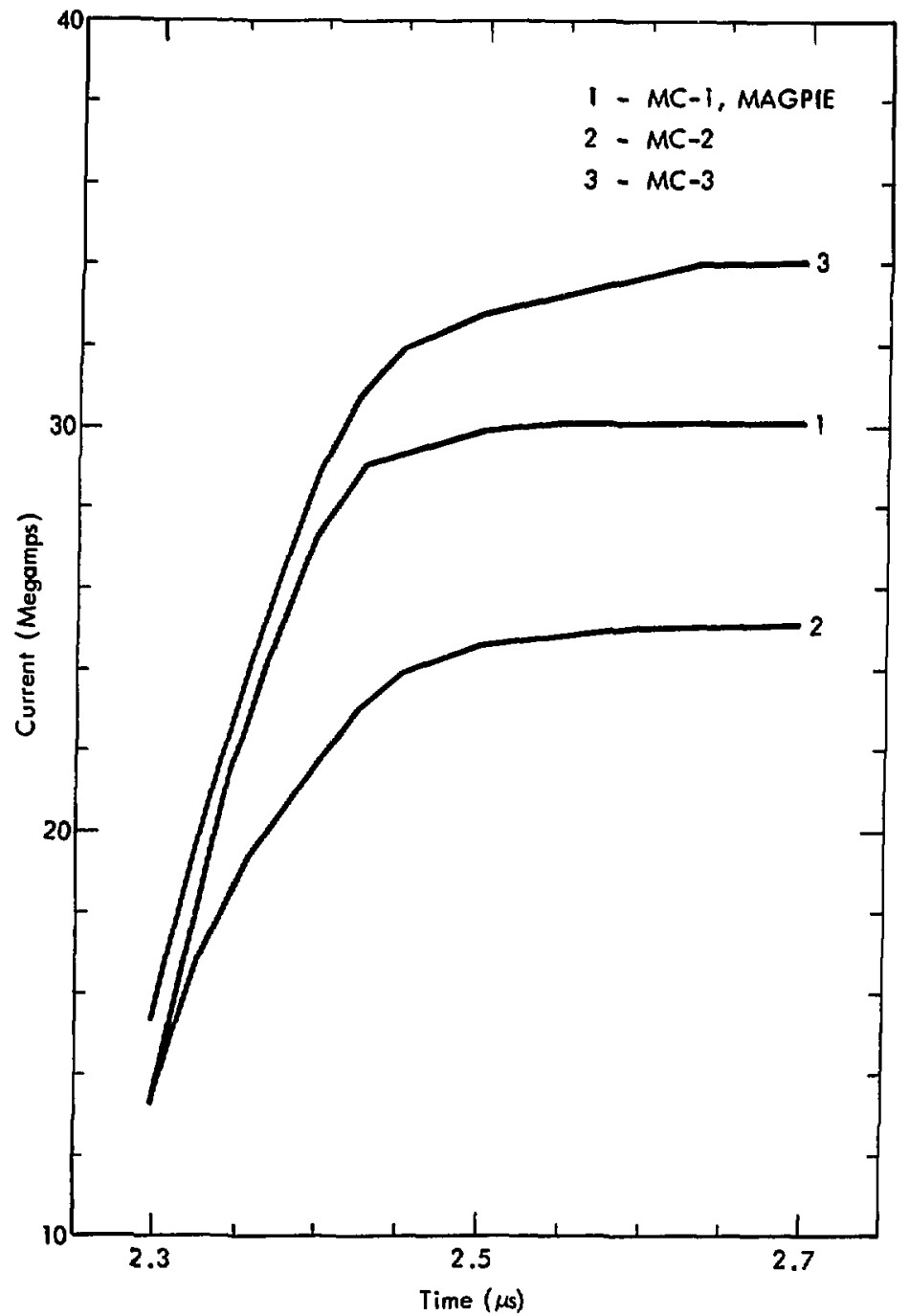

Figure 5.1.1. Current vs time profile for the three MC problems and the one MAGPIE problem. Note current $\# 1$ is for MC- 1 and MAGPIE. 


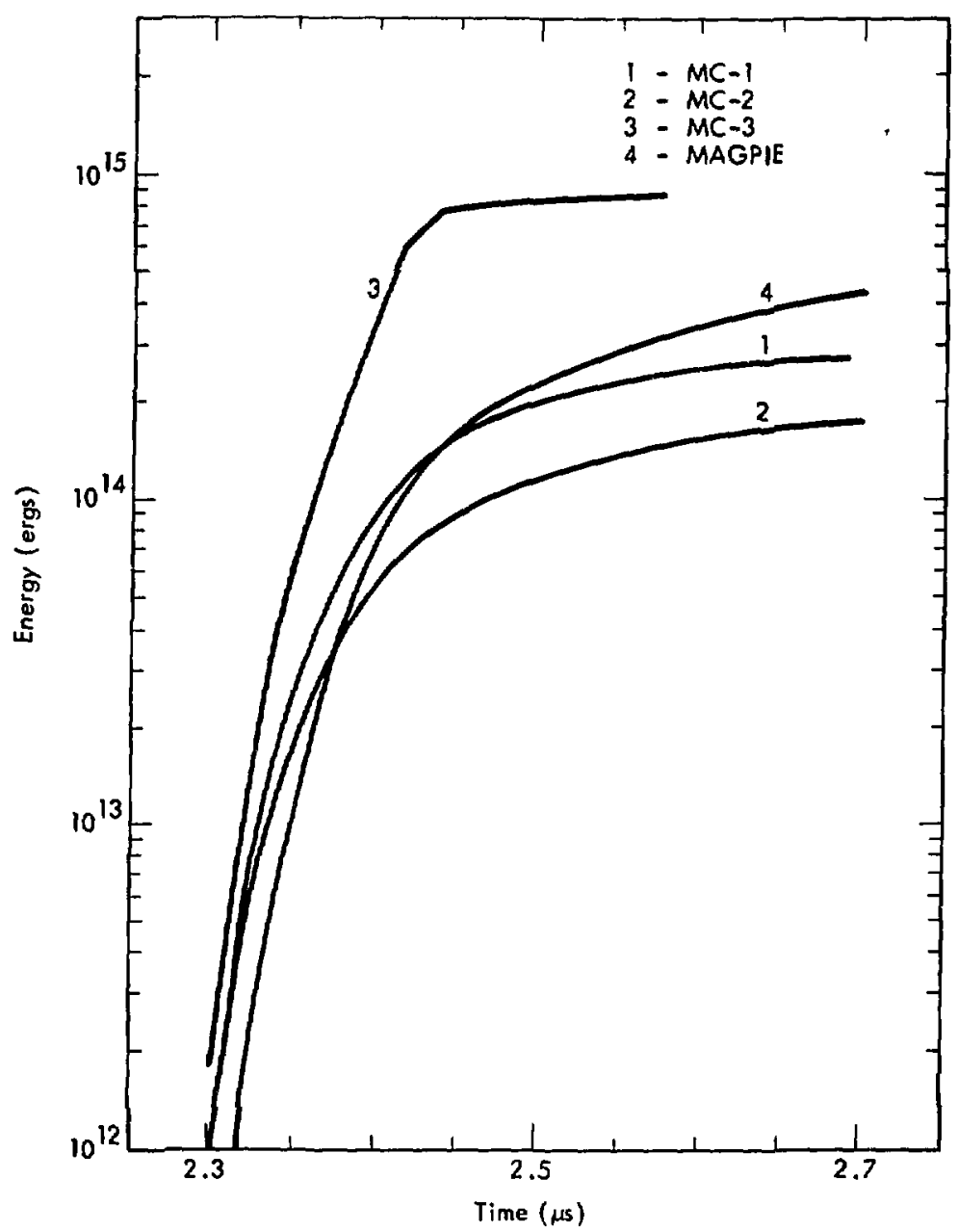

Figure 5.1.2. Alpha energy created from thermonuclear reactions versus tins. 


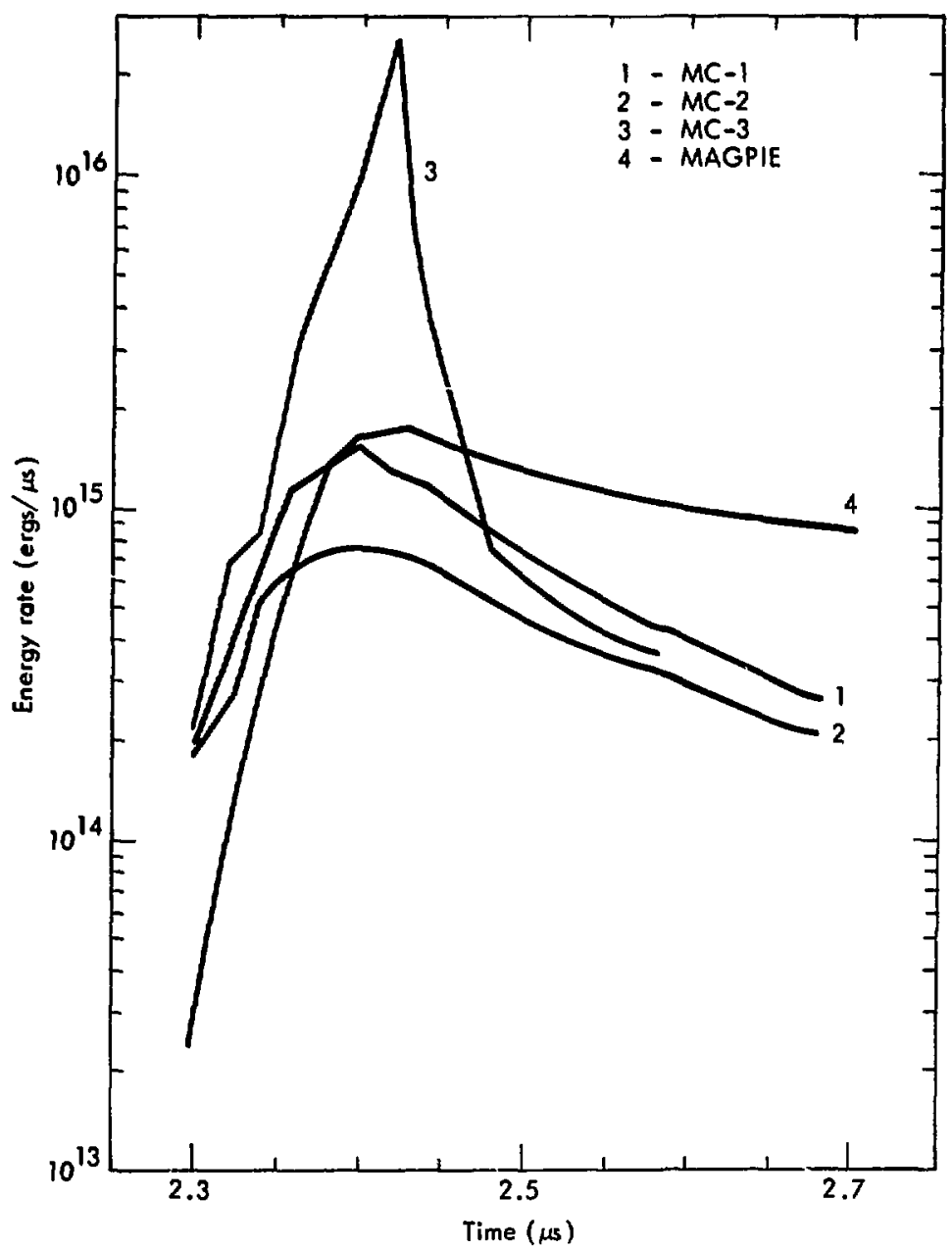

Figure 5.1.3. Rate alpha energy created from thermonuclear reactions versus time. 


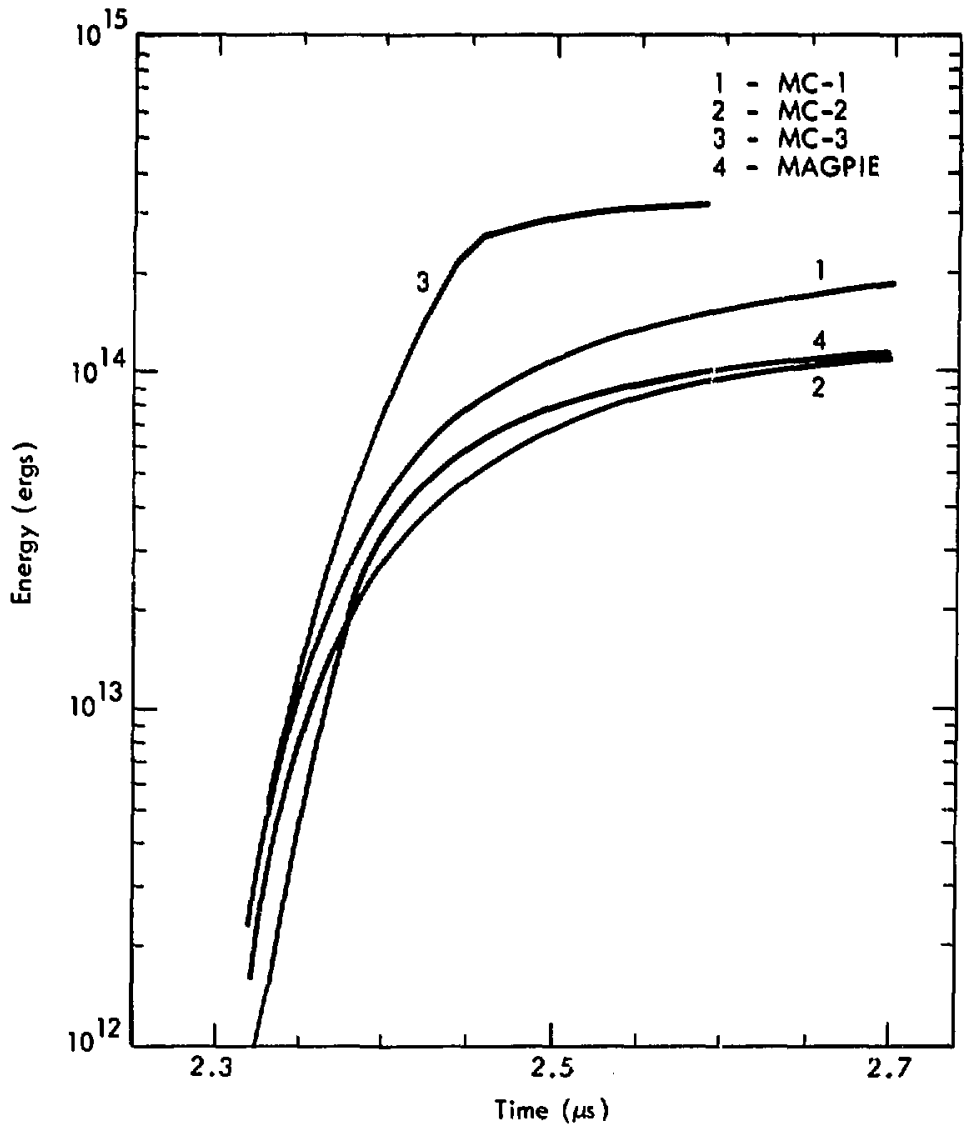

Figure 5.1.4. Alph: energy deposited versus time. 
$-72-$

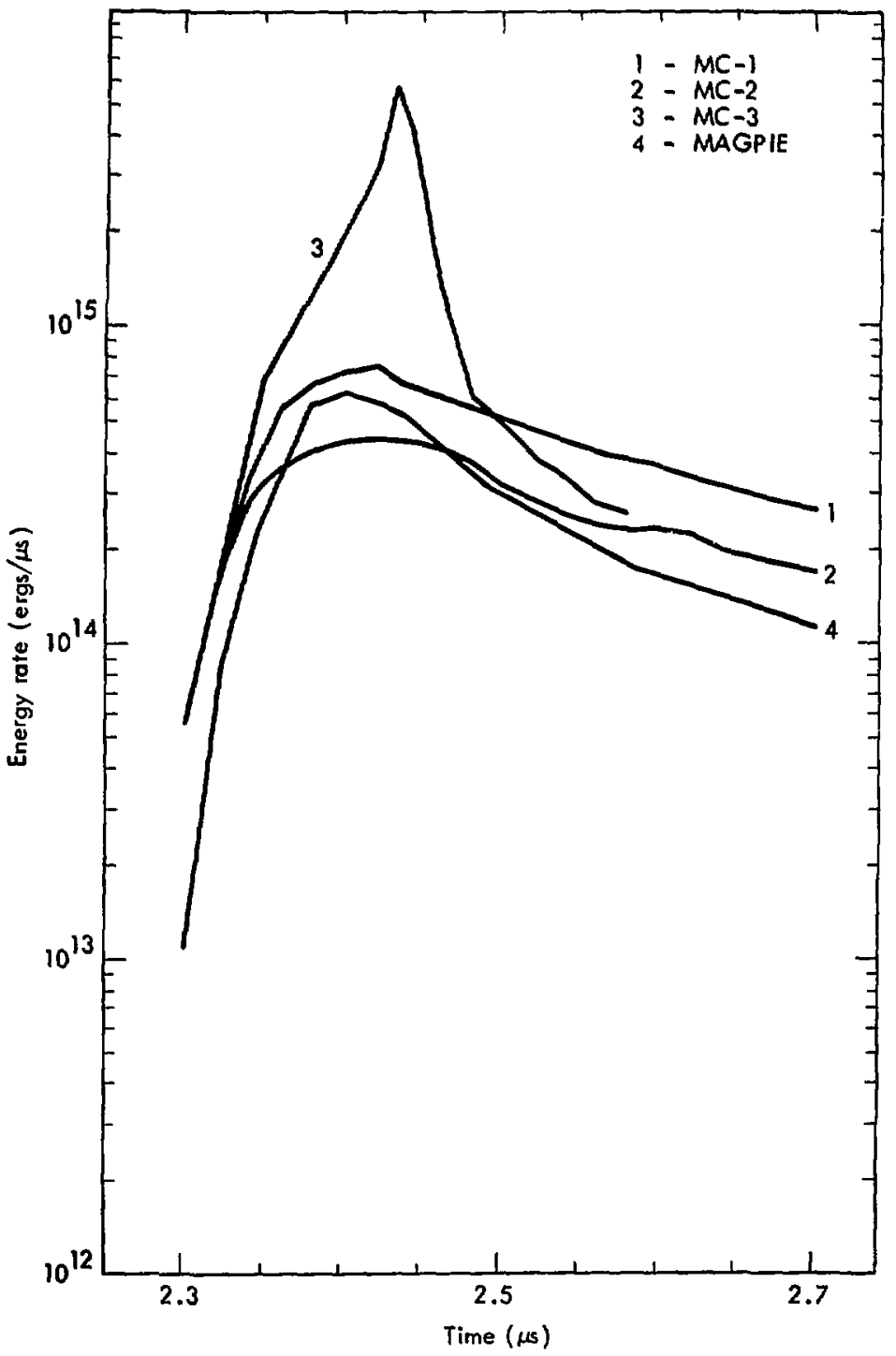

Figure 5.1.5. Rate alpha energy deposited versus time. 
$-73-$

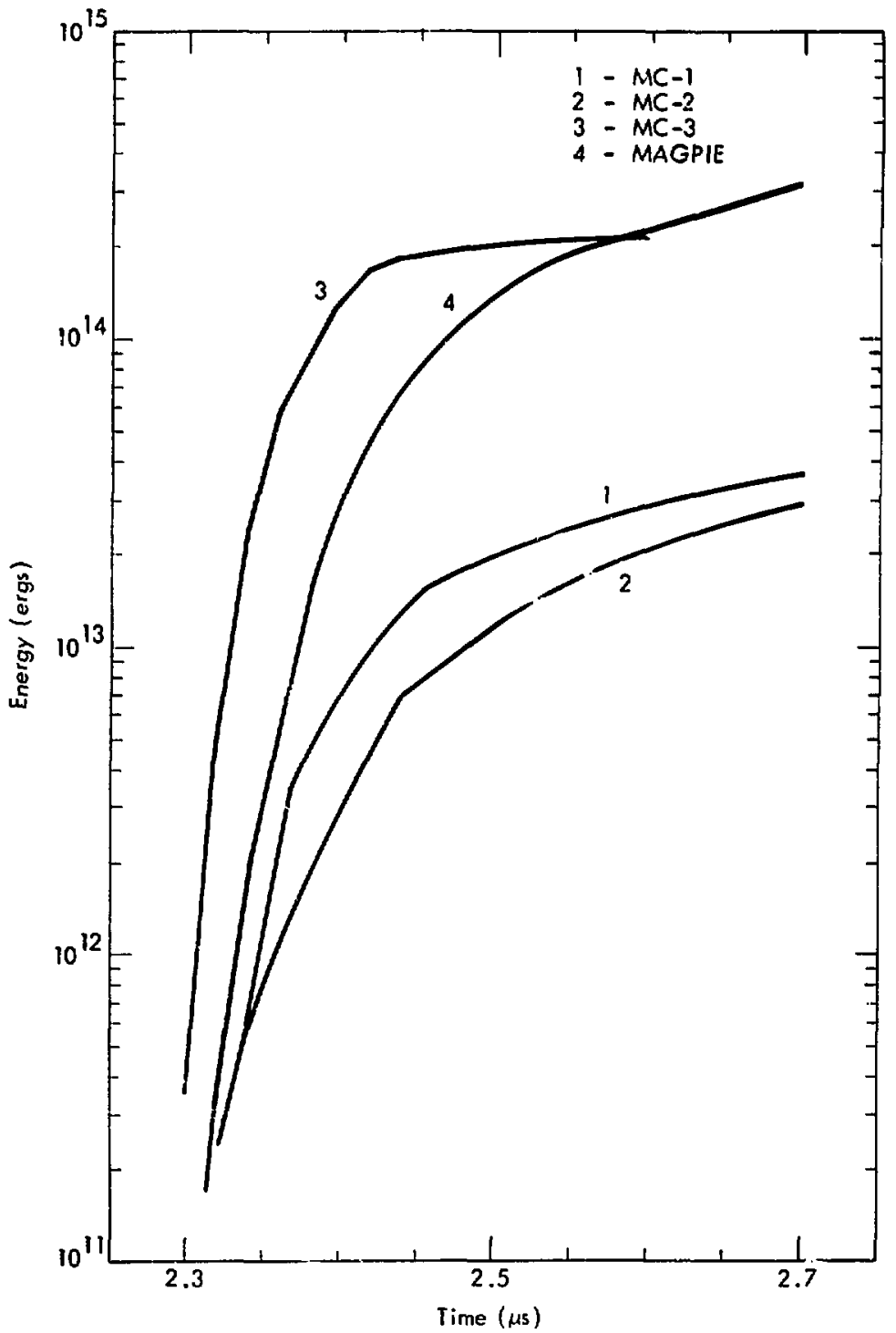

Figure 5.1.6. Energy lost to plasna from drift versus time. 


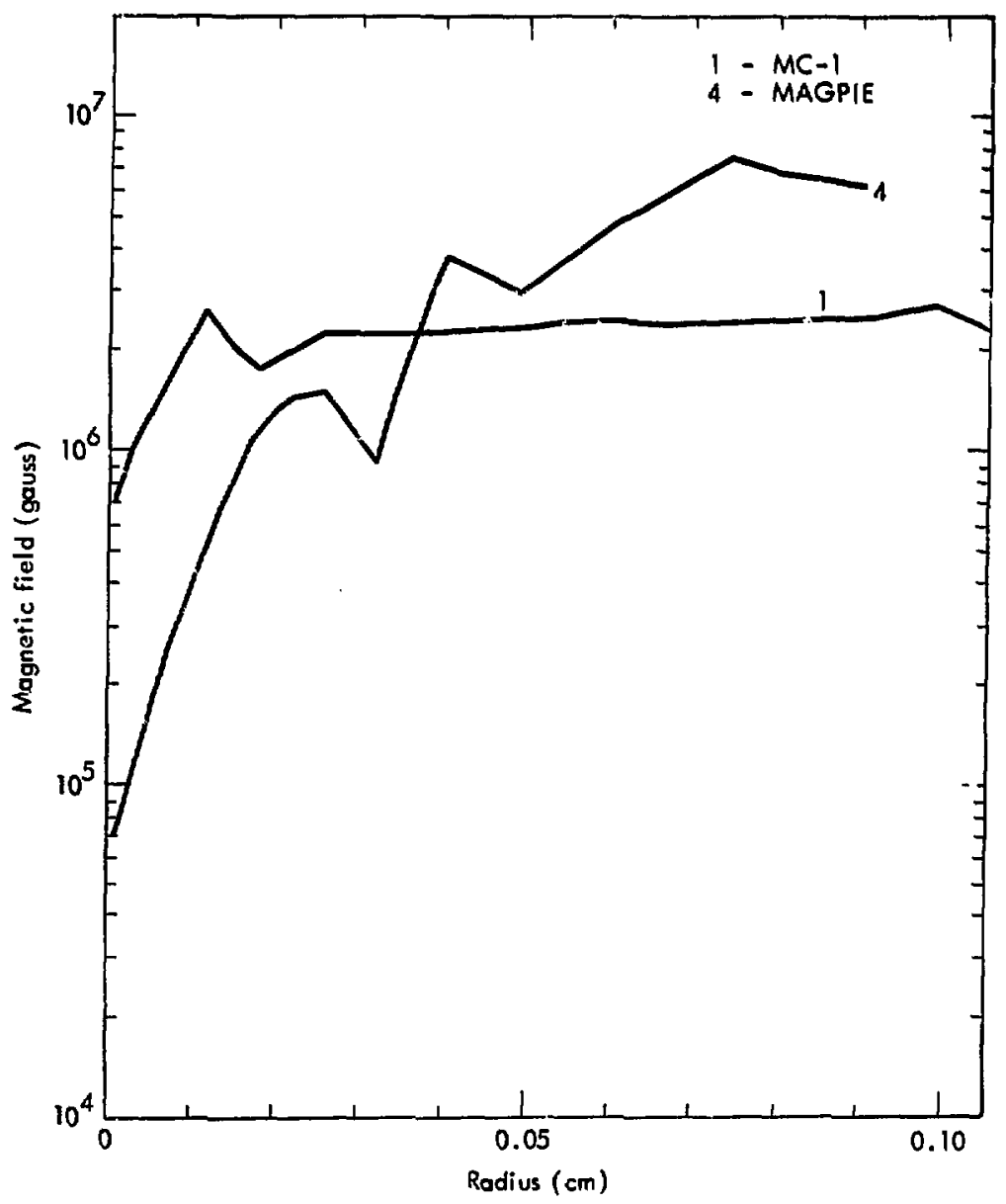

Figure 5.1.7. Magnetic fleld versus radius at $2.6 \mathrm{\mu s}$. 


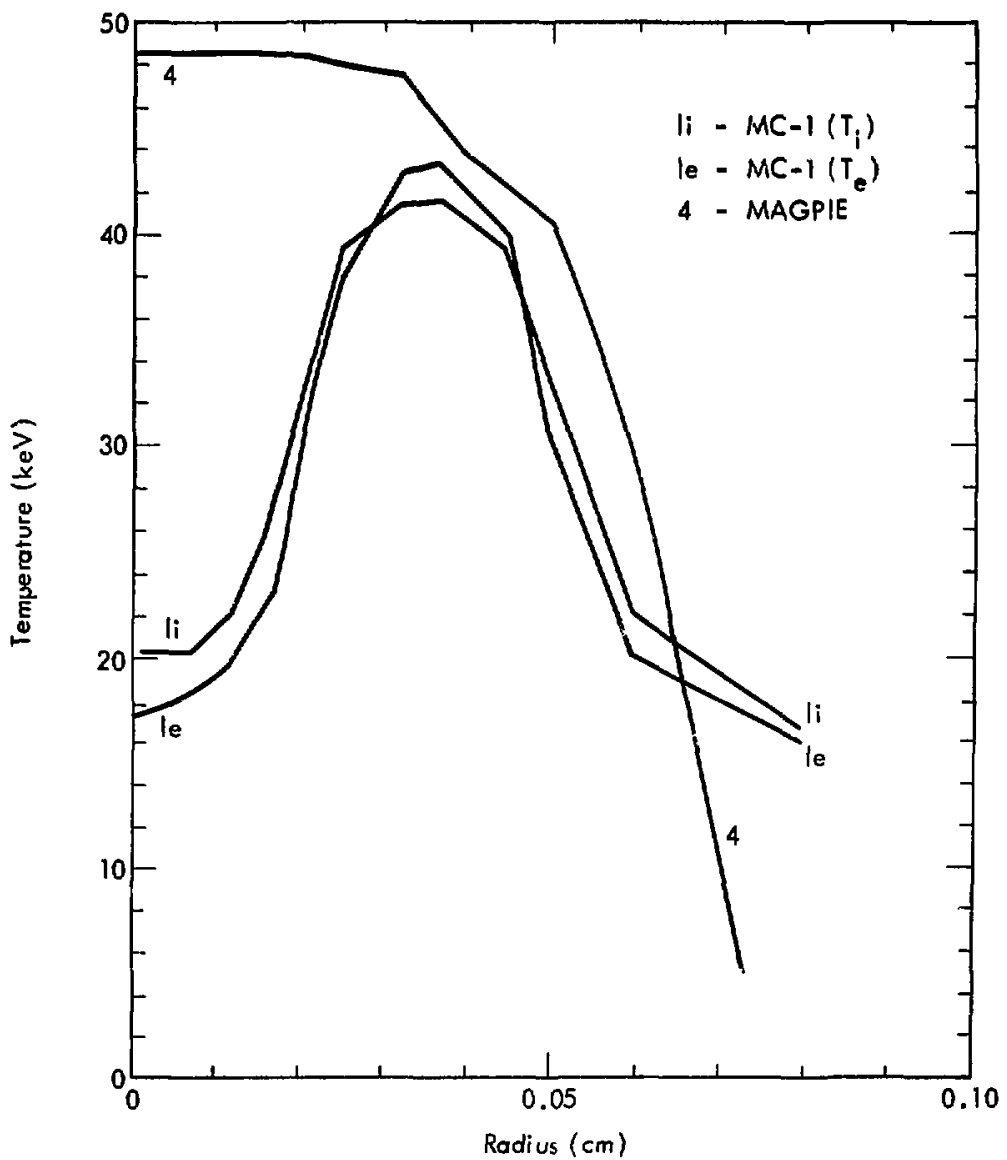

Figure 5.1.8. Temperature versus radius at $2.6 \mu \mathrm{s}$. Note that MAGPIE has only one temperature. 


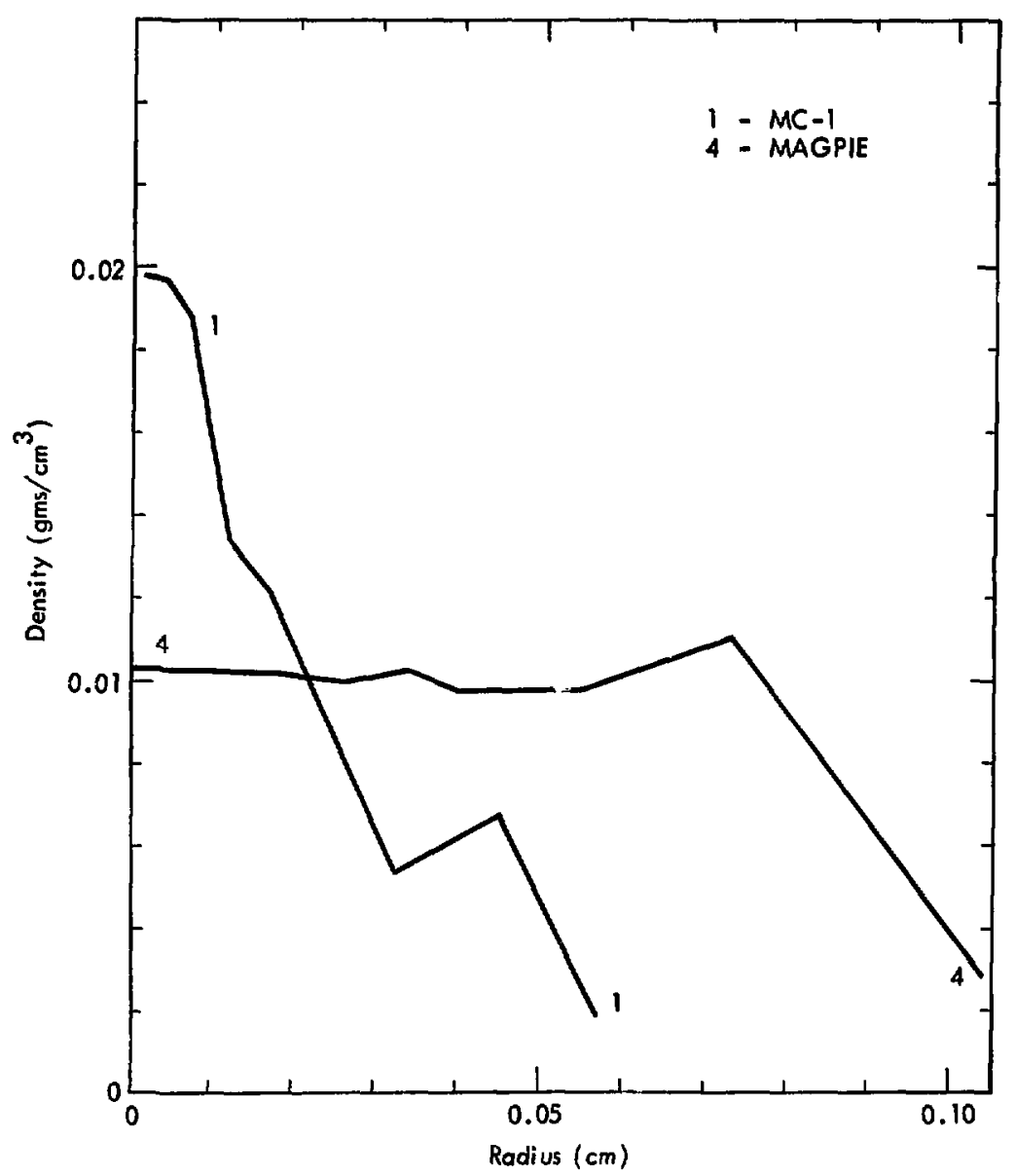

Figure 5.1.9. Density versus radius at 2.6 lis. 


\section{APPENDIX 2: TRANSPORT COEFFICIENTS}

\section{Electric Conductivicy}

From Braginski1, 4 the electric conductivity was calculated from the following formulas

$$
\sigma_{\perp}=\frac{e^{2} n_{e}^{2}}{a_{\perp}} \quad\left\{\sec ^{-1}\right\}
$$

where

$$
a_{1}-\frac{m_{e} n_{e}}{t_{e}}\left(1-\frac{a_{1}^{i} x^{2}+a_{0}^{\prime}}{\Delta_{e}}\right)
$$

and

$$
x=\omega_{e} t_{e}, \Delta_{e}=x^{4}+\delta_{1} x^{2}+\delta_{0} .
$$

The electron collision time is given as

$$
\tau_{e}=\frac{3 \sqrt{m_{e}}\left(K T_{e}\right)^{3 / 2}}{4 \sqrt{2 \pi} e^{4} z^{2} n_{1} \ln \Lambda_{e}}
$$

For $\tau_{e}$ in sec where $K T_{e}$ is given in $\mathrm{keV}$

$$
\tau_{e}=\frac{1.088 \times 10^{10}\left[K T_{e}\{k e v\}\right\}^{3 / 2}}{n_{1} 2^{2} \ln \Lambda_{e}} \quad\{\mathrm{sec}\}
$$

also

$$
\omega_{e}=\frac{e B}{m_{e} c}=1.7588 \times 10^{7} \mathrm{~B}\{\text { gauss }\} \quad\left\{\mathrm{sec}^{-1}\right\}
$$

The coeficients $a_{1}^{\prime}, a_{0}^{\prime}, \delta_{1}, \delta_{0}$ are given in Braginskil in tabular form. They were fitted to polynomials as a functions of $Z$, the atomic number.

$$
a_{1}^{\prime}=4.63+1.786 z^{-1}
$$




$$
\begin{aligned}
& a_{0}^{\prime}=.0678+.4924 z^{-1}+.976 z^{-2}+.3008^{-3} \\
& \delta_{1}=7.482+5.958 z^{-1}+1.35 z^{-2} \\
& \delta_{0}=.0961+.7778 z^{-1}+1.5956 z^{-2}+1.3008 z^{-3}
\end{aligned}
$$

The In $\Lambda_{e}$ term in Eq. (5.2.2) was given by Shkarof $8 k y^{29}$ and Garel1s 30 to be the smaller of the classical expression and the quantum mechanical expression

$$
\begin{aligned}
& \left(\ln \Lambda_{e}\right)_{c l a s s 1 c a l}=33.825+\frac{1}{2} \ln \left[\frac{\left(K T_{e}\right)^{3}}{z^{2} n_{e}}\right] \\
& \left(\ln \Lambda_{e}\right)_{Q . M .}=32.167+\frac{1}{2} \ln \left[\frac{\left(K T_{e}\right)^{2}}{n_{e}}\right]
\end{aligned}
$$

where $\left(K T_{e}\right)$ is expressed in $\mathrm{keV}$, and $\mathrm{n}_{\mathrm{e}}=$ deneity of electrons.

Thus by using the above definition, Eq. (5.2.1) was used to calculate the electric conductivity.

\section{Electron Thermel Conductivity}

Also from Braginakil, the electron thermal conductivity was modeled with the following

$$
\kappa_{1}=\frac{n_{e}\left(K T_{e}\right) \tau_{e}}{m_{e}}=\frac{\left(r_{1}^{\prime} x^{2}+r_{0}^{\prime}\right)}{\Delta_{e}} \quad\left\{\frac{1}{c m-s e c}\right\}
$$

where $\left(\mathrm{KT}_{\mathrm{e}}\right)$ is given in ergs. For $\left(\mathrm{KT}_{\mathrm{e}}\right)$ to be expressed in keV

$$
\kappa_{1}=1.7588 \times 10^{18} n_{e}\left(K_{e}\right) \tau \frac{\left(\gamma_{1}^{\prime} x^{2}+\gamma_{0}^{\prime}\right)}{\Delta_{e}} \quad\left\{\frac{I}{(\mathrm{~cm}-\mathrm{sec}}\right\}
$$


where $\tau_{e}$ is given by Eq. $(5.2 .3)$, and $X$ and $\Delta_{e}$ by Eq. (5.2.2). The coefficients $\gamma_{1}^{\prime}$ and $\gamma_{0}^{\prime}$ were approximated by

$$
\begin{aligned}
& r_{1}^{\prime}=2.5 \\
& r_{0}^{\prime}=10.23 \cdot 9.13 z^{-1}+2.31 z^{-2}
\end{aligned}
$$

\section{Ion Thermal Conductivity}

Again from Braginsk11, the Ion thermal conductivity

$$
K_{1}^{1}=\frac{n_{1}\left(\mathrm{KT}_{1}\right) \tau_{1}}{m_{1}} \frac{\left(2 \mathrm{x}^{2}+2.645\right)}{\Delta_{1}} \quad \frac{1}{\mathrm{~cm}-\mathrm{gec}}
$$

If $\left(K T_{1}\right)$ is to be expressed in $\mathrm{keV}$

$$
\kappa_{\perp}^{1}=9.59 \times 10^{14} \frac{n_{i}\left(\mathrm{KT}_{1}\right) \tau_{1}}{A} \frac{\left(2 \mathrm{x}^{2}+2.645\right)}{\Delta_{1}} \quad \frac{1}{\mathrm{~cm}-\sec } \quad(5.2 .10)
$$

where $\Delta_{1}=x^{4}+2.7 x^{2}+.667$, A is the average atomic mass of the plasma, and $\tau_{1}$, the ion collision time is given by

$$
\tau_{1}=\frac{6.59 \times 10^{11} A^{1 / 2}\left[K T_{1}\right]^{3 / 2}}{n_{1} z^{4} \ln n_{1}} \quad \text { \{sec }
$$

In Eq. $(5,2.11), \mathrm{KT}_{1}$ is also in $\mathrm{keV}$. The varlable $\mathrm{X}$ for lons is $\omega_{1} \tau_{1}$ where $\omega_{1}=9.59 \times 10^{3} \mathrm{zB} / \mathrm{A}\left\{\mathrm{sec}^{-1}\right\}$. The $2 \mathrm{n} \Lambda_{1}$ term, similarly to the In $A_{e}$, is given by the minimum of the following two expressions 29,30

$$
\begin{aligned}
& \left(\ln \Lambda_{1}\right)_{c l a s s i c a l}=33.825+\frac{1}{2} \ln \left[\frac{\left(K T_{e}\right)\left(K T_{1}\right)^{2}}{n_{e} z^{4}}\right] \\
& \left(\ln \Lambda_{1}\right)_{Q . M .}=35.924+\frac{1}{2} \ln \left[\frac{A\left(K T_{e}\right)\left(K T_{i}\right)}{n_{e}}\right]
\end{aligned}
$$

where $\left(K T_{e}\right)$ and $\left(K T_{1}\right)$ are in $\mathrm{keV}$. 
APPENDIX 3: ARTIFICIAL VISCOSITY

When solving MHD differential equations one needs a mechanism to smooth out shock discontinuities. Von Neumann and Richtmyer ${ }^{31}$ developed such a mechanism, an artificial viscosity, denoted by the symbol $q$. Unfortumately, their model doesn't work well in a 2D system, eapecially a 2D Lagrangian system. Schula proposed a tensor viscosity, ${ }^{2}$ or a set of four $q^{1}$ per zone where each $q$ is a measure of the compression along each side of the zone. (See Fig. 5.3.1.) Schulz's equationg for $q$ are

$$
\begin{aligned}
& q^{k}=-c_{0}^{2} \rho\left[\frac{\partial V}{\partial l}\right]^{1 / 2}\left[\frac{\partial}{\partial l}\left(\frac{\partial V}{\partial l}\right)\right]^{3 / 2} \\
& q^{\ell}=-c_{0}^{2} \rho\left[\frac{\partial V}{\partial k}\right]^{1 / 2}\left[\frac{\partial}{\partial k}\left(\frac{\partial V}{\partial k}\right)\right]^{3 / 2}
\end{aligned}
$$

where $q^{\ell}$ is the $q$ along a $k$ line, but differenced with respect to $\ell$; 1.e., $q^{2}$ and $q^{4}$. Also $q^{K}$ is along an $\ell$ line; $q^{1}$ and $q^{3}$. The quantity $V$ Is the speed

$$
V=|\vec{v}|=\left|v_{R} \hat{r}+v_{z} \hat{k}\right|
$$

The partial derivatives are defined as follows

$$
\frac{\partial V}{\partial \ell} \equiv \frac{\vec{x}_{k} \cdot \vec{v}_{\ell}}{\left|\vec{x}_{k}\right|} \quad \frac{\partial V}{\partial k} \equiv \frac{\vec{x}_{\ell} \cdot \vec{v}_{k}}{\left|\vec{x}_{\ell}\right|}
$$

where $\vec{X} \equiv \hat{Z r}-R \hat{k}$, and the subscript refers to differentiation with respect to that variable.

Since the differencing of Eq. (5.3.1), using Eq. (5.3.2) is fairly messy, and also given in detall in Schulz's papers, it will not be repeated here. 


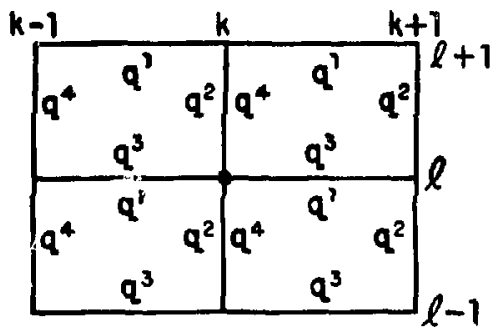

F1gure 5.3.1. Lagranglan zones in $R, Z$ space, showing the position of the censor artificlal viscosity terms. 
APPENDIX 4: ENERGY LOSS RATE EQUATION

The usual starting point for calculating the energy loss (or gain) of an energetic ion to a Maxwellian plasma of density $n_{p}$ is with the equation. $13,29,32,33$

$$
-\frac{d E_{1}}{d t}=\frac{4 \pi n_{p} z_{1}^{2} z_{p}^{2} e^{4} \ln A}{m_{p} v_{1}}\left[\phi-\frac{m_{p}}{\mu} y \phi^{\prime}\right]
$$

where

$$
\begin{aligned}
& \phi=\operatorname{erf}(y)=\frac{2}{\sqrt{\pi}} \int_{0}^{y} e^{-x^{2}} d x \\
& y=\frac{m_{p}}{m_{1}} \frac{E_{1}}{k T_{p}}=\frac{3}{2} \frac{v_{1}^{2}}{\left\langle v_{p}^{2}\right\rangle} \\
& \frac{1}{\mu}=\frac{1}{m_{1}}+\frac{1}{m_{p}}
\end{aligned}
$$

and where the subscript 1 refers to the energetic ion and the subscript $p$ refers to the Maxwellian plasma. These equations will now be analyzed for the specific cases where $v_{1} \gg v_{p}$ and $v_{1} \ll v_{p}$.

A velocity dependent coulomb logarithm by Cooper ${ }^{34}$ was used for In $\Lambda$ in the equations described here.

Case $1\left(v_{1} \ll v_{p}\right)$

If we consider a high energy ion slowing down in a Maxwellian of electrons, then $v_{1} \ll v_{p}$ for cases where the lon is in the low MeV range and the electron temperacure is greater than $1 \mathrm{keV}$. When this is 
$-83-$

the case then $y^{2} \ll 1$ and $\phi$ and $\phi^{\prime}$ from Eq. $(5.4 .1)$ can be simplified.

$$
\phi=\frac{2}{\sqrt{\pi}} \int_{0}^{y} e^{-x^{2}} d x=\frac{2}{\sqrt{\pi}}\left(y-\frac{1}{3} y^{3}\right)
$$

and

$$
y \phi^{\prime}=\frac{2}{\sqrt{\pi}}\left(y-y^{3}\right)
$$

Substituting the above into Eq. (5.4.1) gives

$$
\left(\frac{d E_{1}}{d t}\right)_{e}=\frac{8 \sqrt{\pi} n_{e} z_{1}^{2} e^{4} \ln \Lambda_{e}}{\sqrt{2} m_{1}} \sqrt{\frac{m_{e}}{K T_{e}}}\left(1-\frac{2}{3} \frac{E_{1}}{K T_{e}}\right)
$$

We note that

$$
\left\langle v_{e}\right\rangle=\sqrt{\frac{8 K T_{e}}{\pi m_{e}}}
$$

therefore

$$
\left(\frac{d E_{1}}{d t}\right)_{e}=\frac{16 n_{e} z_{1}^{2} e^{4} \ln \Lambda_{e}}{m_{1}<v_{e}>}\left(1-\frac{2 E_{1}}{3 K T}-\right)
$$

We visually note that for most of the energetic ion's lifetime Eq. (5.4.2) is negative since $E_{1}>\frac{3}{2} \mathrm{KT}_{e}$. When $\mathrm{E}_{1}<\frac{3}{2} \mathrm{KT}$ the elution becomes positive, but the range of the ion once it reaches the elc:tron thermal energy is very small compared to the total range. Therefore since $E_{1}>\frac{3}{2} \mathrm{KT}_{e}$ for most of the ion's range, the factor of 1 in Eq. $(5.4 .2)$ is usually dropped. This results in the form

$$
\left(\frac{d E_{1}}{d t}\right)_{e}=-\frac{32 n_{e} z_{1}^{2} e^{4}}{3 m_{1}\left\langle v_{e}>\right.}\left(\frac{E_{1}}{k T}\right) \ln \Lambda_{e}
$$


Case $2\left(v_{1} \gg v_{p}\right)$

If we consider a high energy lon slowing down in a Maxwellian of lons then $v_{1} \gg v_{p}$ for most of the range. In this case $y^{2} \gg 1$, therefore the bracketed part of Eq. $(5.4 .1)$ becomes

$$
\phi-\frac{m_{1}}{\mu} y \phi^{\prime}=\frac{2}{\sqrt{\pi}} \int_{0}^{y} e^{-x^{2}} d x-\left(1+\frac{m_{1}}{m_{1}}\right) y c^{-y^{2}}
$$

In the limit as $y \rightarrow \infty$, the above goes to 1 . Therefore

$$
\left(\frac{d E_{1}}{d t}\right)_{i}=-\frac{4 \pi n_{1} z_{1}^{2} z_{i}^{2} e^{4} \ln \Lambda_{1}}{m_{i} v_{1}}
$$

Th1s is the destred form of the equation for a sigle ion fluid Maxwellian. For a multi fon fluid

$$
\left(\frac{d E_{1}}{d t}\right)_{1}=-\frac{4 \pi e^{4} z_{1}^{2}}{v_{1}} \ln \Lambda_{1} \sum_{i=1}^{N} \frac{n_{1} z_{1}^{2}}{m_{1}}
$$

where $\mathrm{N}$ is the total number of Maxwellian fon species.

The total energy loss to the energetfc ion is just the sum of Eqs. (5.4.3) and (5.4.5).

$$
\frac{d E_{1}}{d t}=\left(\frac{d E_{1}}{d t}\right)_{e}+\sum_{1=1}^{N}\left(\frac{d E_{1}}{d t}\right)_{1}
$$

Equation (5.4.6) can now be integrated to obtain the energy losses to the Monte Carlo ion bundles due to the small angle coulomb collisions (Section 3.2). 
APPENDIX 5: TRACKING IN A 2D LAGRANGIAN CYLINDRICAL MESH

In doing particle simulations one needs to push a particle through a coordinate mesh. As explained in Section 3.2, given a $\Delta t$ one can calculate a new velocity $\vec{v}$ and a new position $\vec{k}$ for a particle provided one knows the equation of motion. The tracking of a particle in a $2 D$ Lagrangian (quadrilateral) mesh was progranmed in cartesian coordinates, hence the elemental distance $(\Delta \vec{X})$ a particle moves in some given $\Delta t$ is a straight line, 1.e., the curved orbital path of the ion is composed of many short straight line segments. Also since variableg change from zone to zone it is necessary to guarantee that a chosen $\Delta t$ does not carry a particle beyond the boundarles of the zone it was in. Instead a particle is advanced fust to the boundary and the zonal parameters in the equation of motion are changed accordingly. Then the particle is allowed to enter the new zone and concinue on tcs way. The scheme used and about to be described was modeled from one proposed by the late CaIvin Gardner ${ }^{35}$.

Two important calculations must be made to do the tracking (particle pushing): first a coordinate transformation is made on the velocity vector to simplify the tracking and second, the distance to the zone boundary is calculated. Doing the coordinate transformation also grearly simplifies the distance to boundary calculation.

The Monte Carlo bundle has a cartesian 3D velocity and position.

$$
\begin{aligned}
& \vec{x}=x_{0} \hat{i}+y_{0} \hat{j}+z_{0} \hat{k} \\
& \vec{v}=v_{x} \hat{i}+v_{y} \hat{j}+v_{z} \hat{k}
\end{aligned}
$$


but because of cylindrical symetry a coordinate transformation can be performed which essentially reduces the velocity to two dimensions. Figure 5.5.1a shows the projection of $\vec{v}$ in the $R-2$ plane and Fig. 5.5.1b ahows the projection of $\vec{v}$ in a constant 2 plane ( $x, y$ plane). Since there is no $\theta$ dependence in the varlables, the cylinder can be rotated such that $v_{x}$ goes to zero and $v_{y}$ is positive; Fig 5.5.1c. The motion 18 now confined to a $y-z$ plane provided the track is linear, which it is for each elemental $\Delta \vec{X}$. At the end of each $\Delta \vec{X}$ a particle will deflect out of the $y-z$ plane due to a rotation from the Lorentz force or a collision. Therefore a coordinate rotation is done after each time step while tracking each particle. After rotation the particle has a new position and velocity

$$
\begin{aligned}
& \vec{x}^{\prime}=x_{0}^{\prime} \hat{i}+y_{0}^{\prime} \hat{j}+z_{0}^{\prime} \hat{k} \\
& \vec{v}^{\prime}=v_{y}^{\prime} \hat{j}+v_{z} \hat{k}
\end{aligned}
$$

The rotation is done using the following procedure.

$$
\begin{aligned}
& \alpha=\frac{v_{x}}{v} ; \beta=\frac{v_{y}}{v}, \gamma=\frac{v_{z}}{v} \\
& \alpha^{\prime}=0 ; \gamma^{\prime} * \gamma ; B^{\prime}=\sqrt{1-\gamma^{2}} \\
& x_{n}^{\prime}=\left(\beta x_{0}-\alpha y_{0}\right) / B^{\prime} \\
& y_{0}^{\prime}=\left(\alpha x_{0}+\beta y_{0}\right) / B^{\prime} \\
& z_{0}^{\prime}=z_{0} ; v_{x}^{\prime}=0 \\
& v_{y}^{\prime}=\beta^{\prime} v ; v_{z}^{\prime}=v_{z}
\end{aligned}
$$


where $a, B$, and $\gamma$ are the usual direction cosines and the prime superscript refers to the new coordinate frame. Note that $v$, the speed, remains constant in the rotation.

The problem now is to calculate the distance to boundary along the stralght line trajectory of the velocity vactor in thin $y-z$ plane.

The straight line segments in the R-Z plane that define a Lagrangian zone are really conic sections in the cylinder - see Fig. 5.5.2a. From Fig. 5.5.2a, b, $c$, it can be seen that the intersection of the surface generated by the line 1-2 and a y-z plane is a curved Iine -- a hyperbola. Hence the distance to boundary calculation is the intersection of the velocity vector projection with a hyperbola. Since there are four sides to a zone and the particle doesn't know whIch one 1 t will hit, one must look at the intersection of the velocity vector with the 4 hyperboles generated by the 4 zone sides.

Without going into the algebra, ine distance to boundary for one of the four sides is given by

$$
R=\frac{S K+\frac{\beta^{\prime}}{Y^{\prime}} y_{0}^{\prime} \pm \sqrt{\left[s^{2}-\left(\frac{\beta^{\prime}}{\gamma^{\prime}}\right)^{2}\right] x_{0}^{\prime 2}+\left[\frac{\beta^{\prime}}{\gamma^{\prime}} K+S y_{0}^{\prime}\right]^{2}}}{r^{\prime}\left[s^{2}-\left(\frac{\beta^{\prime}}{\gamma^{\prime}}\right)^{2}\right]}
$$

where

$$
S=\frac{R_{1}-R_{2}}{z_{1}-z_{2}} \text { and } K=S\left(z_{2}-z_{0}^{\prime}\right)-R_{2}
$$

and where $\left(R_{1}, z_{1}\right),\left(R_{2}, z_{2}\right)$ are the cylindrical coordinates of a Lagranglan line element. This equation is a quadratic and ylelds two solutions, and 
since this calculation must be done for all four sides of the zone, eight solutions for are obtained. The smallest, positive, real solution 18 the valid one.

Having calculated the distance tg boundary, the lon bundle can be advanced In the $y_{-2}$ plane the appropriate $\Delta \frac{}{\mathrm{X}}$-- the distance to boundary or the distance calculated from the lurentz force for the specified $\Delta t$, whichever is gmaller. 


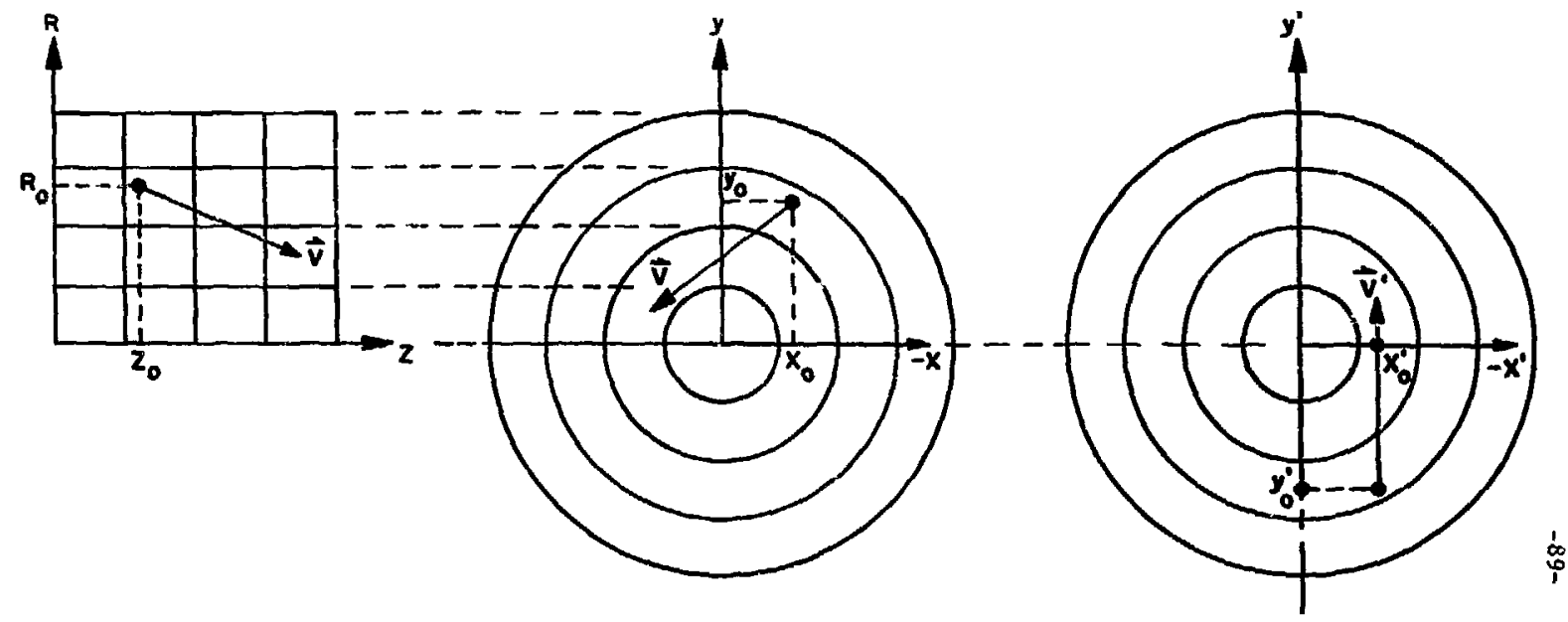
$5.5 .1 a$
3.5 .16
$5.5 .1 c$

Higure 5.5.1a,b,c. A particle velocity vector in cyilndri:ai coordinates (Fig. a,b); and after a ccordinate transformation $(\mathrm{FI} \xi . \mathrm{c})$. 


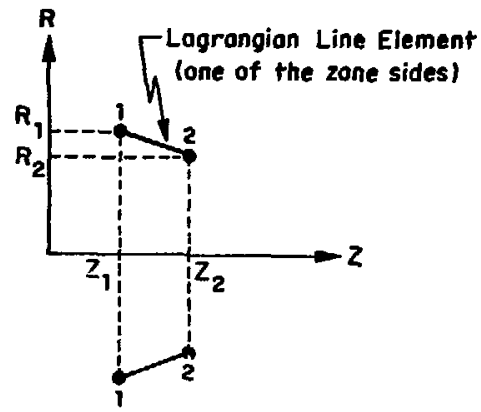

$5.5 \cdot 2 a$

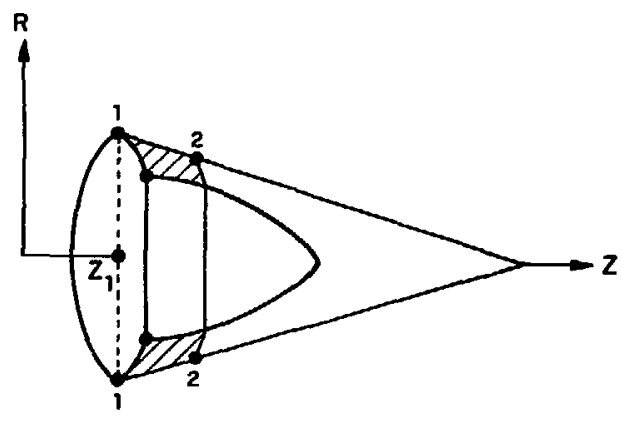

$5.5 .2 b$

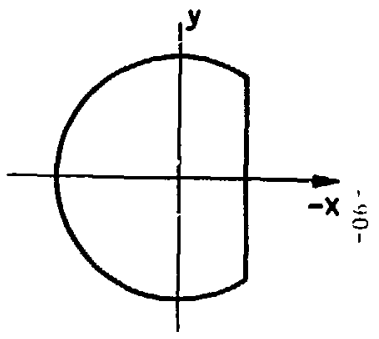

$5.5 .2 c$

Figure -.5.2a,b,c. A Lagrangian line element (FIg. a) rerresents a crnic sur jace of revolution (Fig. b). The intersection or a $y-7$ plare with the conic surface is a hyperbola ( 


\section{APPENDIX 6: MONTE CARLO TECHNIQUES}

A rough outline of the Monte Carlo process might be summarized as follows:

1. A source of non-Maxwellian energetic lens must exist in the phys.cal system being simulated.

2. This source of ions 18 subdivided into $\mathrm{N}$ bundleg, where $\mathrm{N}$ is called the sample size. The determination of $\mathrm{N}$ will be discussed below.

3. The bundles are given an initial asition, veloctey vector, etc., that completely describe the bundle.

4. Bundies are now tracked and suffer collisions for a serles of minor $\Delta t^{\prime} s$ until a major $\Delta t$ is reached. At the end of the major $\Delta t$, the particle is stored away on disc (census). Another bundle is now tracked. When all bundles have been tracked for the major $\Delta t$, the code advances the other pieces of physics (NHD) for the major $\Delta t$. When this is completed a new major $\Delta t$ is started; new source particles may be generated, and the census particles are retrieved for further tracking.

A critically important parameter in running any Monte Carlo calculation is the determination of $N$. It must be large enough such that the cumulative history of the bundles is representative of the problem, but small enough to be practical to run. In this model $N$ was determined automatically within the code and was constantly varying depending on the source strength.

$$
N=\text { Integer part of }\left\{\frac{E_{g}}{E_{0}}\right\}
$$


where $E_{S}$ is the source energy and $E_{0}$ is an input parameter. In other words $E_{0}$ is approximately the energy of the Monte Carlo bundles to be tracked. The wejght is the actual number of ions within the bundle. A simple example may help.

Suppose in an acsua: source we had $10^{20}$ alpha particles, each of $2 \mathrm{MeV}$ energy. The total energy of the source is thus $2 \times 10^{20} \mathrm{MeV}$. Now suppose the user decides that he doesn't want Monte Carlo bundles roaming around his problem with energies grenter than $10^{17}$ NeV. Then he would set $E_{0}=10^{17}$ and $N$ would be calculated to be 2000 ; $1 . e$, the code would track 2000 bundies, each of $10^{17} \mathrm{MeV}$. Since the weight is the actual number of alphas in the bundle

$$
W T=\frac{\text { bundle energy }}{\text { ion energy }}=\frac{10^{17} \mathrm{MeV}}{2 \mathrm{MeV}}=5 \times 10^{16}
$$

The sample size was determined in this way to avoid having very energetic bundles traveling through the mesh with the potential to wreak havoc with a small zone. Also $\mathrm{X}$ is a function of the source; you don't want to generate a large $N$ when the source is negligible or off. If the source is substantial and if $N$ is small, then it is quite possible to have bundles (generated in a large zone) that are larger in mass and energy than another zone. And if this bundle should interact with this small zone, then tha entire energy and mass of the small zone could be depleted -r causing disaster. Normally one would set $E_{0}$ to be equal to some fraction of the internal energy of the smallest zone. Thus all bundles would be assured of being small enough to not create too much trouble. 
There is one problem with this approach. If $E_{0}$ is based upon the smallest zone, and if there are also many large zones, then one ends up with a very large number of small bundles. This problem is easily overcome by spliteing bundles when they pass into small zones, or randomly throwing out bundles when they pass into large zunes (Russian Roulette). When playing Russian Koulette, one must multiply the curviving bundles weight and energy by all approprtate iactor to conserve cnergs, mass and momentum. It Is only conserved on the nverige.

Becauge the cillculation of $A$ was a function of $E_{s}$. the sample s1:c can grow without buund as the source grows. But machine limitations force an upper maximun of $x$ to be approximately $j \times 10^{5}$ for a time step. Practically thoupir, a "reasonable" value of $N$ ffor an entire mesh -- over a single $\Delta t$ ) would be approximately $5 \times 10^{4}$ bundles. a CDC 7600 computer was able to process this many butndles for a single major time step in one to two minutes of CPU time. Sonte Carlo can be expensive!

There are obviously lots of ways one may deternine a sarple size criteria for a Nonte Carlo calculation, but this method seemed to be fairly simple and trouble free. Of course what it bolls down to is that one wants a sample size that is somewhere between the reglons of nonsense ( $N$ too sma11) and non-affordability (N too large). 
APPEXOX 7: CXERGETLC loB SOLRCES FOR NORTL CARLO NODEL

The energet ic ions of the Monte Carlo model puy come from onc or frons several progranaed sources, dopending on the problem. Une may generato the problen with the fons already in the problem, In other words, the particles may exist within the probleri when the problem is started. Hother suurce may be an externally injected beam of neutralizad lons. Hy neutrallech, 1: is meant that the fon bear belng infected into the pismi has frec companfon glectrons traveling; along, with tt. The fon beam description is supplied by the user, where aly lon of $\therefore=2$ may bo spectfled, it is not necessary to go into details, Lue in a sonte carlo nodel it is quite easy to build very complicated ion beams; such as an anisutropic, sileed fsotope, time and energ: dependent seuree. A third source a Sonte Carto partfeles is one that has already bea moncienca; bhe seatcered carget particles irom a Stonce Carlo event (sca section 3.3). Ihis is a secondary source, in that it takes other Yonce Garlo ;articles to create ther.

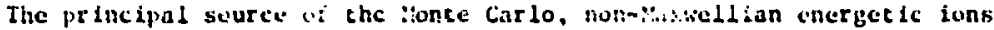
modeled was the therronuclear reaction products of aburilng Stikwellian rlasma, Ncconsodations were made to simulate the following reactions

$$
\begin{aligned}
& d+d-3_{l: c}+n \\
& d+d-t+p \\
& d+t \rightarrow a+n \\
& d+3 \text { He } \rightarrow a+p
\end{aligned}
$$


knowing the Mxwellian averaged cross sections, the ion temperature $\left(I_{i}\right)$ and the fon densities, the reaction rates (R) are simply calculated between the $1^{\text {th }}$ and $j^{\text {th }}$ species

$$
k_{1 j}=n_{i} n_{j} \text { covs }{ }_{i-j}
$$

and when $1=1$

$$
R_{11}=\frac{1}{2} n_{i}^{2} \text { covs } i-1
$$

The Mavell avcraged eross sections were tabulated from Greene ${ }^{5}$, as a function of $I_{f}$. Hultiplying $E q .(5.7 .5)$ by a zone's volume and a time step gives the actual number of reactions that took place, and the actud number of the reaction products in that zone. These reaction products are then groupud into represcntative bundles, given an appropriate energy, a weight, isotroplc scatcering angles, and a random initial position within the zone. Now they are Nonte Carlo bundles of energatic tons and the tracking can proceed. 


\section{ACKNOWLEDCNENTS}

There were so nany people who helped me in this study that it is difficult to eloquently thank them, Nany thanks have to go my advisors James $\%$. Shearer and John kllleen for their guidance and instruction; to Willian D. Schulz ior his untiring willingness to listen and give advice; to George B. Zimnerman for his NHD notes and for finding that elusive "bug"; and to lrvin $R$. Lindemuth for the enlightening conversations on MHD (and fishing).

Special thanks go to Edward Garelis, who more than anyone, has helped me make it through the past 5 years of school; to Rex L. Evans, an incredible programmer who has saved me many hours of agony with his patient assistance; to Susan Flynn for typing, and typlng, and typling, and .....; and to my supervisors Will lam P. Crowley and William A. Lokke for their support.

Most importantly, a very special note of chanks goes to my wife Kay who has always encouraged me, and has $r$ aver complained about the ordeal that I have put her through. And finally I want to thank the two people who helped get me started -- my parents. 


\section{REFERENCES}

1. Methods in Computation Physics, B. Alder, S. Fernback, and N. Rotenberg, editors (Academic Press, New York, 1970) Vol. 9.

2. W. D. Schulz, "Two-Dimensional Lagranglan Hydrodnamic Difference Equationg", In Mechods in Computational Physics, B. Alder, S. Fernbach, and M. Rotenberg, editore (Academic Press, New York, 1964), Vol. 3, p. 1.

3. W. D. Schulz, Two-Dimensional Lagrangian Hydrodynamic Difference. Equations, Lawrence Livermore Laboratory Rept., UCRL-6776 (1963).

4. S. I. Braginsk1i, "Iransport Processes in a Plasma", In Reviews of Plasma Physics, M. A. Leontovish, editor (Consultants Bureau, New York, 1965), Vo1, 1, p. 205.

5. S, L. Greene, Jr., Maxwe11 Averaged Cross Sections for Some ThermoNuclear Reactlons on Light Isotopes, Lawrence Livermore Laboratory Repc., UCRL-70522 (1967).

6. H. Alfven and E. Smars, Nature, 188, 801 (1960).

7. J. Shearer, "Gas Embedded 2-Pinch: New Approach to an 01d Fusion Concept", Energy and Technology Review, Lawrence Livermore Laboratory Rept. UCRL-52000-75-6 (June 1975).

8. S. Glassione and R. H. Lovberg, Concrolled Thermonuclear Reactlons (Van Nostrand Relnhold Co., New York, 1960).

9. K. Nishimura, "Velocity Dependence of the Coulomb Logarithm and the Temperature Equilization of Electrons and Ions In a Plasma", The Physical Review 152 (1966), 177-181. 
10. W. Schulz, Lawrence Livermore Laboratory, Livermore, California, private communication (1974).

11. G. Zimmerman, Lawrence LIvermore laburatory, Livermore, Cal1fornia, unpublished notes on LASNEX code (1974).

12. T. 13. St1x, "Heating of Toroldal plnsmis by Neutral injection", Plasma Phystes 14, 367 (1972).

13. L. Spitzer, Jr. Physics of fully lonlzed gases, 2nd edition (Interscience Publishers, New York, 1967).

14. E. G. Corman, W. E. Lowwe, G. E. Cooper and A. M. Kinslow, Nultigroup Diffusion of Energetic Charged Particles, Lawrence Livermore Laboratory Rept., UCRL-75870 Rev. I (1975), (prepared for submission to Nuclear Fusion).

15. J. W. Shearer, Ion Beam Compression of Thermonuclear Pellets, Lawrence Liveruore Laboratory Rept., UCRL-75619-Rev. 1 (i975), (to be published in iuclear fusion).

16. E. G. Cormin, Elastic Nuclear Scatterlng of Deuterons and Alphas in Deuterlum, Lawrence Livermore Laburatory Rept., tCID-15971 (1971).

17. E. G. Corman, Lawrence Livermorc Laboratury, Livermore, California, private communication and unpublished notes (1974).

18. Slain, et al., Phys. Rev., 74, 1594 (1948).

19. Wilson, et a.l, Nicl. Phys., A126, 193 (1969).

20. Brolley, et al., Phys. Rev., 117, 1307 (1960). 
21. Blair, et a1., Plyys. Rev., 75, 1680 (1949).

22. Galonsky, et al., Phys. Rev., 98, 586 (1955).

23. W. L. Kruer, Plasma Sinulation Using Parclcle Codes, Lawrence Livermore Laboratory Rept., UCKL-75995 (1974), (prepared for submiasion to Nuclear Technology).

24. R. D. Evans, The Atomic Nucleus (NcGraw H111 Book Co., Inc., New York, 1955).

25. T. H. Johnson, Guiding-Center Stmulation of Toroidai Plamas, Lawrence Livermore Laboratory Rept. UCRL-51725 (1974), (Ph.D. Thesis, Graduste Division of the University of California, Davis).

26. C. H. Hartman, D. Y. Cheng, J. Eddleman, R. H. Muıger, J. W. Shearer, and $K$. Struve, Studies of the Ges-Imbedded Z-PInch, Proceedings of the Topical Conference on Pulsed High Beta Plasmas (paper B2-4) LKAEA Culham Laboratory, 1975.

27. C. W. Hartman, D, Y. Cheng, G, E. Cooper, J. L. Eddleman, and H. Munger, High Density Fusion and the Z-Pinsh, Proceedings of the Fifth Conference on Plasma Physics and Controlled Nuclear Fusion Research, IAEA, Tokyo, p, 653, 1974.

28. D. Steinberg, R. E. Kiddur, and A. B, Cectl, A One-Dinensional Nagnetohydrodynamlcs Code, Lawrence Livermore Laboratory Rept., UCRL-14931 (1966).

29. I. P. Shkarofsky, T. W. Johnston and M. P. Bachynsk1, The Particle Kinetlcs of Plasmas (Addison-Wesley Publishing Co., Reading, Massachusetts, 1966).

30. E. Garelis, Lawrence Livermore Laboratory, Livermore, California, unpublished notes (1974). 
31. J, von Neumann and R. D. Richtmyer, "A Vethod for the Numerical Calculation of tiydrodynamic Shocks", J. Appl. Phys. 21 (1950), 232-237.

32. S. Chandrasekhar, "Il.e Time of Relaxition of Stellar systems", Astrophysical Journal 93 (1941), 285-304.

33. S. I. Butler and M. J. Buckingham, "Energy Loss of a Fast Ion In a Plasma", The lhysical keview 126, 1 (1962).

34. G. E. Cooper and F. T. Hermin, "lffect of Velocity Dependence of Coulomb Logarithin on the Solution of the Fokker-Planck Equation", The Hhysics of Fluids 16 (1973), 118-121.

35. C. Gardner, Lawrence Livermore Laboratory, Livermore, California, unpublished notes (1969). 